\title{
Bogomolov's inequality for Higgs sheaves in positive characteristic
}

\author{
Adrian Langer
}

Received: 8 November 2013 / Accepted: 20 June 2014 / Published online: 11 July 2014 (C) The Author(s) 2014. This article is published with open access at Springerlink.com

\begin{abstract}
We prove Bogomolov's inequality for Higgs sheaves on varieties in positive characteristic $p$ that can be lifted modulo $p^{2}$. This implies the Miyaoka-Yau inequality on surfaces of non-negative Kodaira dimension liftable modulo $p^{2}$. This result is a strong version of Shepherd-Barron's conjecture. Our inequality also gives the first algebraic proof of Bogomolov's inequality for Higgs sheaves in characteristic zero, solving the problem posed by Narasimhan.
\end{abstract}

\section{Mathematics Subject Classification (2010) $\quad 14 \mathrm{G} 17 \cdot 14 \mathrm{~J} 60 \cdot 14 \mathrm{~F} 05$}

\section{Introduction}

Let $X$ be a smooth projective variety of dimension $n \geq 2$ defined over an algebraically closed field $k$. Let us fix an ample divisor $H$ on $X$. In [3] Bogomolov proved a celebrated inequality saying that in case $n=2$ and $k=\mathbb{C}$, the discriminant $\Delta(E)=2 r c_{2}(E)-(r-1) c_{1}^{2}(E)$ of any slope $H$-semistable rank $r$ vector bundle $E$ is non-negative. Together with the Mehta-Ramanathan restriction theorem this implies that for $k=\mathbb{C}$ and any $n \geq 2$ we have $\Delta(E) H^{n-2} \geq 0$ for any slope $H$-semistable torsion free sheaf $E$ of rank $r$ on $X$.

In [21] we proved that in positive characteristic the same inequality holds for strongly $H$-semistable sheaves. On the other hand, it is well known that

\footnotetext{
A. Langer $(\bowtie)$

Institute of Mathematics, University of Warsaw, ul. Banacha 2, 02-097 Warsaw, Poland e-mail: alan@mimuw.edu.pl
} 
in general this inequality fails. For example, any counterexample to Kodaira's vanishing on surfaces (see [30,33]) leads to a slope $H$-semistable vector bundle violating Bogomolov's inequality. However, we prove the following surprising theorem:

Theorem 1 Assume that $X$ can be lifted to the ring $W_{2}(k)$ of Witt vectors of length 2. Then for any slope $H$-semistable sheaf $E$ of rank $r \leq p=$ char $k$ we have

$$
\Delta(E) H^{n-2} \geq 0
$$

This theorem was known only in the surface case for rank 2 vector bundles in characteristic 2 (see [34, Corollary 11]). Let us recall that such an inequality has many applications, for example to adjoint linear systems via Reider's method (see [2, IV, Section 11] and Sect. 7).

In case of complex varieties there exist several proofs of Bogomolov's inequality. Roughly, there are three different proofs of this inequality. The first one, due to Bogomolov (see $[3,12]$ ), is algebraic, using the fact that symmetric powers of a semistable bundle have few sections. The second one, due to Gieseker (see [11]), is also algebraic, using reduction to positive characteristic and studying sections of the Frobenius pull back of the bundle. The third proof is analytic, using the Kobayashi-Hitchin correspondence.

Unfortunately, none of these proofs works in positive characteristic and we do not know how to prove the above theorem without proving a much stronger result for Higgs bundles.

Let us recall that one of the starting points of Simpson's work on nonabelian Hodge theory was his paper [35] in which he proved the generalized Kobayashi-Hitchin correspondence: on a compact complex Kähler manifold every stable Higgs bundle has a Hermitian-Yang-Mills metric. As a corollary, Simpson proved Bogomolov's inequality for stable Higgs bundles on such manifolds. This inequality played an important role in further development of the subject (see, e.g., $[28,29]$ ).

To the author one of the most intriguing and eluding problems was how to prove Bogomolov's inequality for Higgs sheaves using algebraic methods. This problem was posed by Narasimhan in late 1990s. Early attempts to solve this problem worked only in low rank and used vanishing theorems to reduce the problem to the usual Bogomolov's inequality (see $[1,20])$. The first algebraic approach to Bogomolov's inequality via bounding the number of sections of symmetric powers does not seem to work in the Higgs case. This motivated the author to work on the positive characteristic case and resulted in a proof of Bogomolov's inequality for strongly semistable sheaves (see [21]). Unfortunately, this did not shed light on the original problem. 
An algebraic proof of Bogomolov's inequality for Higgs sheaves became possible only after the appearance of Ogus and Vologodsky's non-abelian Hodge theory in positive characteristic (see [31]). We also use in a crucial way the results of [21] to prove a weak version of Bogomolov's inequality for semistable modules with generalized connections. Bogomolov's inequality for Higgs sheaves in characteristic zero is then proven by reducing to positive characteristic and applying the above inequality to the vector bundle with a connection corresponding to the Higgs bundle. This argument needs a small adjustment as Ogus and Vologodsky prove Simpson's correspondence only for Higgs bundles satisfying some nilpotence conditions which are not satisfied in our case.

But the real main aim of this paper is to establish the following Bogomolov's inequality for Higgs sheaves in positive characteristic:

Theorem 2 Assume that $X$ be can be lifted to the ring $W_{2}(k)$ of Witt vectors of length 2. Then for any slope $H$-semistable Higgs sheaf $(E, \theta)$ of rank $r \leq p$ we have

$$
\Delta(E) H^{n-2} \geq 0
$$

The strategy of proof of the above theorem is the following. First we use the results of [21] to prove a weak version of Bogomolov's inequality for vector bundles with generalized connections (see Theorem 6). Then we deform $(E, \theta)$ to a slope semistable system of Hodge sheaves and we recall a construction of a Higgs-de Rham sequence for such systems. Existence of such sequences for semistable Higgs sheaves was conjectured by Lan et al. in [18] and we prove their conjecture in a separate paper (see [25]; after this paper was submitted there also appeared another proof of this result in [17]).

The sequence is constructed by means of Ogus and Vologodsky's inverse Cartier transform. This transform changes a slope semistable system of Hodge sheaves into a slope semistable coherent sheaf with an integrable connection with "larger Chern classes". Then we can deform this sheaf into another slope semistable system of Hodge sheaves and we repeat the process constructing a sequence of slope semistable systems of Hodge bundles. If the discriminant of the original sheaf were negative then this process would make the discriminants of further terms in this sequence more and more negative. But by the above weak Bogomolov's inequality, the discriminants in the constructed sequence are bounded below, so we get a contradiction.

In [3, Theorem 5] Bogomolov proved that on a complex projective surface of general type we have $4 c_{2} \geq c_{1}^{2}$. Later, Miyaoka [27] and Yau [38], proved the optimal inequality $3 c_{2} \geq c_{1}^{2}$. It is well known that both these inequalities fail in positive characteristic (see, e.g., [7, 15,30,33,37]). In 1989 Shepherd-Barron conjectured (see [34, p. 244]) that the crucial theorem in algebraic proofs of the 
Bogomolov-Miyaoka-Yau inequality (see [3, Theorem 4] and [27, Theorem 2"]) holds for surfaces liftable to $W_{2}(k)$. We recall an unpublished example of Ekedahl et al. (see [8, p. 23, Remark ii]) showing that this conjecture is false. It is therefore very surprising that in characteristic $p \geq 3$ the Miyaoka-Yau inequality $3 c_{2} \geq c_{1}^{2}$ holds for surfaces of non-negative Kodaira dimension liftable to $W_{2}(k)$ (see Theorem 13). This is a strong version of the inequality conjectured by Shepherd-Barron (see [34, p. 244]). In characteristic 2 we reprove the weaker inequality $4 c_{2} \geq c_{1}^{2}$ known from [34].

Another interesting result says that semistable Higgs sheaves with vanishing Chern classes are locally free (see Theorem 11). The analogous result [36, Theorem 2] was proven by Simpson using analytic methods and the (topological) Lefschetz hyperplane theorem. We obtain his result as a corollary by purely algebraic methods.

Other applications of Theorem 2 include an effective restriction theorem for Higgs sheaves (see Theorem 10) and an analogue in positive characteristic of the new restriction theorem of Bruzzo and Hernández Ruipérez [5] (see Theorem 12). As a corollary, we also obtain a new proof of the original theorem in characteristic zero.

In the final section we give a quick proof by Bhargav Bhatt of the fact that Frobenius split varieties are liftable to the ring of Witt vectors of length 2. In particular, all the obtained results are valid for sheaves on such varieties.

For simplicity, we deal in this paper only with the usual Higgs sheaves. However, very similar methods work also for sheaves with $\lambda$-connections and in case of logarithmic Higgs bundles. The parabolic version is more complicated and requires a quite heavy notation. All these generalizations will be treated elsewhere.

The paper is organized as follows. In Sect. 2 we gather several preliminary results. Section 3 contains proofs of various Bogomolov type inequalities. In Sect. 4 we apply these inequalities to prove restriction theorems for Higgs sheaves. Then in Sect. 5 we study semistable Higgs sheaves with vanishing Chern classes. In Sect. 6 we prove the Bogomolov-Miyaoka-Yau inequality in positive characteristic. In Sect. 7 we quickly recall applications of Bogomolov's inequality to adjoint linear systems. In Sect. 8 we prove that Frobenius split varieties are liftable to $W_{2}$.

\subsection{Notation}

If $x$ is a real number then we set $[x]_{+}:=\max (x, 0)$.

Let $S$ be a scheme of characteristic $p$ (i.e., $\mathscr{O}_{S}$ is an $\mathbb{F}_{p}$-algebra). By $F_{S}^{r}: S \rightarrow S$ we denote the $r$-th absolute Frobenius morphism of $S$ which corresponds to the $p^{r}$-th power mapping on $\mathscr{O}_{S}$. If $X$ is an $S$-scheme, we denote by $X^{\prime}$ the fiber product of $X$ and $S$ over the first absolute Frobenius 
morphism of $S$. The absolute Frobenius morphism of $X$ induces the relative Frobenius morphism $F_{X / S}: X \rightarrow X^{\prime}$.

For a rank $r$ torsion free sheaf $E$ on a smooth projective variety $X$ we set

$$
\Delta(E):=2 r c_{2}(E)-(r-1) c_{1}(E)^{2} .
$$

\section{Preliminaries}

\subsection{Semistability}

Let $k$ be an algebraically closed field of any characteristic. Let $X$ be a smooth projective variety of dimension $n \geq 1$ over $k$ and let $D_{1}, \ldots, D_{n-1}$ be nef divisors on $X$ such that the 1-cycle $D_{1} \ldots D_{n-1}$ is numerically nontrivial, i.e., there exists a divisor $D$ such that $D D_{1} \ldots D_{n-1} \neq 0$.

Let $E$ be a rank $r$ torsion free sheaf on $X$. We define the slope of $E$ by

$$
\mu(E)=\mu_{D_{1}, \ldots, D_{n-1}}(E)=\frac{c_{1}(E) D_{1} \ldots D_{n-1}}{r} .
$$

We say that $E$ is slope $\left(D_{1}, \ldots, D_{n-1}\right)$-semistable if for every subsheaf $E^{\prime} \subset$ $E$ we have

$$
\mu_{D_{1}, \ldots, D_{n-1}}\left(E^{\prime}\right) \leq \mu_{D_{1}, \ldots, D_{n-1}}(E) .
$$

If $H$ is an ample divisor then we say that $E$ is slope $H$-semistable, if it is slope $(H, \ldots, H)$-semistable.

For simplicity, in the following we usually omit in notation dependence of slopes on the collection $\left(D_{1}, \ldots, D_{n-1}\right)$. The maximal (minimal) slope in the Harder-Narasimhan filtration of $E$ (with respect to $\left(D_{1}, \ldots, D_{n-1}\right)$ ) is denoted by $\mu_{\max }(E)\left(\mu_{\min }(E)\right.$, respectively). If $k$ has positive characteristic then we set

$$
L_{\max }(E)=\lim _{m \rightarrow \infty} \frac{\mu_{\max }\left(\left(F_{X}^{m}\right)^{*} E\right)}{p^{m}}
$$

and

$$
L_{\min }(E)=\lim _{m \rightarrow \infty} \frac{\mu_{\min }\left(\left(F_{X}^{m}\right)^{*} E\right)}{p^{m}} .
$$

By [21, Theorem 2.7] $L_{\max }(E)$ and $L_{\min }(E)$ are well defined rational numbers.

We say that $E$ is strongly slope semistable if all the Frobenius pull backs $\left(F_{X}^{m}\right)^{*} E$ of $E$ for $m \geq 0$ are slope semistable. This is equivalent to saying that $L_{\max }(E)=\mu(E)$ (or, equivalently, to $L_{\min }(E)=\mu(E)$ ). 


\subsection{Higgs sheaves}

A Higgs sheaf $(E, \theta)$ is a pair consisting of a coherent $\mathscr{O}_{X}$-module $E$ and an $\mathscr{O}_{X}$-linear map $\theta: E \rightarrow E \otimes_{\mathscr{O}_{X}} \Omega_{X}$ satisfying the integrability condition $\theta \wedge \theta=0$.

We say that $(E, \theta)$ is slope semistable if the inequality $\mu\left(E^{\prime}\right) \leq \mu(E)$ is satisfied for every Higgs subsheaf $\left(E^{\prime}, \theta^{\prime}\right)$ of $(E, \theta)$.

A system of Hodge sheaves is a Higgs sheaf $(E, \theta)$ with decomposition $E=\bigoplus E^{j}$ such that $\theta: E^{j} \rightarrow E^{j-1} \otimes_{\mathscr{O}_{X}} \Omega_{X}$.

As above, a system of Hodge sheaves $(E, \theta)$ is slope semistable if the inequality $\mu\left(E^{\prime}\right) \leq \mu(E)$ is satisfied for every subsystem of Hodge sheaves $\left(E^{\prime}, \theta^{\prime}\right) \subset(E, \theta)$. It is easy to check that then $(E, \theta)$ is slope semistable as a Higgs sheaf (see [25, Corollary 3.5]).

Using properness of the Hitchin morphism one can show the following lemma (see [25, Corollary 5.7]):

Lemma 1 Let $(E, \theta)$ be a torsion free, slope semistable Higgs sheaf on $X$. Then there exists an $\mathbb{A}^{1}$-flat family of Higgs sheaves $(\tilde{E}, \tilde{\theta})$ on $X \times \mathbb{A}^{1}$ such that for any closed $k$-point $t \in \mathbb{A}^{1}-\{0\}$ the restriction $\left(\tilde{E}_{t}, \tilde{\theta}_{t}\right)$ to the fiber over $t$ is isomorphic to $(E, \theta)$ and $\left(\tilde{E}_{0}, \tilde{\theta}_{0}\right)$ is a slope semistable system of Hodge sheaves.

Let $(E, \nabla)$ be a coherent sheaf with an integrable connection. Similarly to the above, one can define slope semistability for coherent sheaves with integrable connections. In [25, Theorem 5.5] (see also [17, Theorem 2.2]) we prove the following theorem:

Theorem 3 If $(E, \nabla)$ is slope semistable then there exists a canonical Griffiths transverse filtration $N^{0}=0 \subset N^{1} \subset \cdots \subset N^{m}=E$ such that the associated graded system of Hodge sheaves is slope semistable. This filtration is preserved by the automorphisms of $(E, \nabla)$.

The canonical filtration $N^{\bullet}$ from Theorem 3 is called Simpson's filtration of $(E, \nabla)$ and denoted by $N_{S}^{\bullet}$.

\subsection{Simpson's correspondence in positive characteristic}

In this subsection we recall the main results of Ogus and Vologodsky [31].

Let $f: X \rightarrow S$ be a morphism of schemes in characteristic $p$. A lifting of $X / S$ modulo $p^{2}$ is a morphism $\tilde{X} \rightarrow \tilde{S}$ of flat $\mathbb{Z} / p^{2} \mathbb{Z}$-schemes such that $X \rightarrow S$ is equal to the base change of $\tilde{X} \rightarrow \tilde{S}$ by the closed embedding $S \rightarrow \tilde{S}$ defined by $p$.

Let $\mathrm{MIC}_{p-1}(X / S)$ be the category of $\mathscr{O}_{X}$-modules with an integrable connection whose $p$-curvature is nilpotent of level less or equal to $p-1$. Similarly, 
let $\mathrm{HIG}_{p-1}(X / S)$ denote the category of Higgs $\mathscr{O}_{X^{\prime}}$-modules with a nilpotent Higgs field of level less or equal to $p-1$. The following theorem is one of the main results of Ogus and Vologodsky (see [31, Theorem 2.8]):

Theorem 4 Let $\mathscr{X} / \mathscr{S}:=\left(X / S, \tilde{X}^{\prime} / \tilde{S}\right)$ be a smooth morphism with a lifting $\tilde{X}^{\prime} / \tilde{S}$ of $X^{\prime} / S$ modulo $p^{2}$. Then the Cartier transform

$$
C_{\mathscr{X} / \mathscr{S}}: \operatorname{MIC}_{p-1}(X / S) \rightarrow \mathrm{HIG}_{p-1}\left(X^{\prime} / S\right)
$$

defines an equivalence of categories with quasi-inverse

$$
C_{\mathscr{X} / \mathscr{S}}^{-1}: \operatorname{HIG}_{p-1}(X / S) \rightarrow \operatorname{MIC}_{p-1}\left(X^{\prime} / S\right) .
$$

A small variant of the following lemma can be found in proof of [31, Theorem 4.17]:

Lemma 2 Let $(E, \theta) \in \mathrm{HIG}_{p-1}\left(X^{\prime} / S\right)$. Then

$$
\left[C_{\mathscr{X} / \mathscr{S}}^{-1}(E)\right]=F_{X / S}^{*}[E],
$$

where [.] denotes the class of a coherent $\mathscr{O}_{X}$-module in Grothendieck's $K$ group $K_{0}(X)$.

Proof By assumption there exists a filtration $N^{m}=0 \subset N^{m-1} \subset \cdots \subset$ $N^{0}=(E, \theta)$ of length $m<p$ by Higgs subsheaves such that $N_{i}=N^{i} / N^{i-1}$ has zero Higgs field. Then $C_{\mathscr{X} / \mathscr{S}}^{-1}\left(N_{i}\right)=F_{X / S}^{*} N_{i}$ and therefore

$$
\left[C_{\mathscr{X} / \mathscr{S}}^{-1}(E)\right]=\sum\left[C_{\mathscr{X} / \mathscr{S}}^{-1}\left(N_{i}\right)\right]=\sum\left[F_{X / S}^{*} N_{i}\right]=F_{X / S}^{*}[E] .
$$

As a corollary to Theorem 4 and Lemma 2 we get the following:

Corollary 1 Assume that $S$ is the spectrum of an algebraically closed field and $X$ is projective. Let $(E, \theta)$ be a torsion free Higgs sheaf with nilpotent Higgs field of level less than $p$. Then it is slope semistable if and only if the corresponding sheaf with integrable connection $C_{\mathscr{X} / \mathscr{S}}^{-1}(E, \theta)$ is slope semistable.

Proof Let $\left(V^{\prime}, \nabla^{\prime}\right) \subset(V, \nabla)=C_{\mathscr{X} / \mathscr{S}}^{-1}(E, \theta)$. Then by Theorem $4(E, \theta) \simeq$ $C_{\mathscr{X} / \mathscr{S}}(V, \nabla)$ contains $\left(E^{\prime}, \theta^{\prime}\right) \simeq C_{\mathscr{X} / \mathscr{S}}\left(V^{\prime}, \nabla^{\prime}\right)$. So if $(E, \theta)$ is slope semistable then by Lemma 2 we have

$$
\mu\left(V^{\prime}\right)=p \mu\left(E^{\prime}\right) \leq p \mu(E)=\mu(V),
$$

which proves slope semistability of $C_{\mathscr{X} / \mathscr{S}}^{-1}(E, \theta)$.

Implication in the opposite direction is completely analogous. 


\subsection{Higgs-de Rham sequences}

Let $k$ be an algebraically closed field of characteristic $p>0$. Let $X$ be a smooth projective $k$-variety of dimension $n$ that can be lifted to a smooth scheme over the ring $W_{2}(k)$ of Witt vectors of length 2 . Let us set $S=$ Spec $k$ and $\tilde{S}=\operatorname{Spec} W_{2}(k)$. Since $X^{\prime}$ is isomorphic to $X$, this provides the lifting needed in Ogus' and Vologodsky's version of Simpson's correspondence.

Let $(E, \theta)$ be a rank $r$ torsion free Higgs sheaf with nilpotent Higgs field. Let us assume that $r \leq p$ so that level of nilpotence of $(E, \theta)$ is less than $p$. The following definition is taken from [18]:

Definition 1 A Higgs-de Rham sequence of $(E, \theta)$ is an infinite sequence

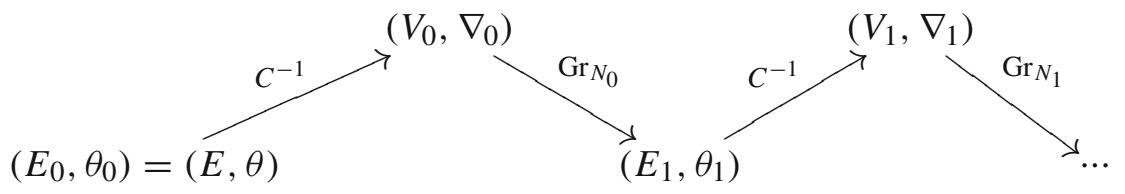

in which $C^{-1}=C_{\mathscr{X} / S}^{-1}$ is the inverse Cartier transform, $N_{i}^{\bullet}$ is a Griffiths transverse filtration of $\left(V_{i}, \nabla_{i}\right)$ and $\left(E_{i+1}:=\operatorname{Gr}_{N_{i}}\left(V_{i}\right), \theta_{i+1}\right)$ is the associated Higgs sheaf.

Let us note the following lemma:

Lemma 3 If in a Higgs-de Rham sequence of $(E, \theta)$ there exists $i$ such that $E_{i}$ is locally free then $E_{j}$ and $V_{j}$ are locally free for $j \leq i$.

Proof Note that local freeness of $V_{i}$ follows from the construction of the inverse Cartier transform. Indeed, locally $V_{i}$ is isomorphic to the Frobenius pull back of $E_{i}$ (see [31, Theorem 2.8,3]), so it is locally free.

Now note that there exists a family whose general member is isomorphic to $V_{i-1}$ and the special one is $E_{i}$. By openness of local freeness we see that $V_{i-1}$ is locally free. At this point one can also use the following argument due to the referee: if a sheaf admits a finite exhaustive filtration whose associated graded is locally free, then it is locally free (proof by induction on the length of the filtration). Therefore if $E_{i}$ is locally free then $V_{i-1}$ is locally free.

This implies that $E_{i-1}$ is locally free by descent of flatness under the Frobenius morphism (again we use this locally using the construction of the inverse Cartier transform). This last fact is a special case of Ferrand's theorem (see [10]). Now the assertions follow by induction on decreasing $j$.

The following theorem was conjectured by Lan-Sheng-Zuo [18, Conjecture 2.8] and it follows from Theorem 3 (see [25, Theorem 5.12]; see also [17, Theorem 3.2] for a slightly weaker statement): 
Theorem 5 If $(E, \theta)$ is slope semistable then there exists a canonically defined Higgs-de Rham sequence

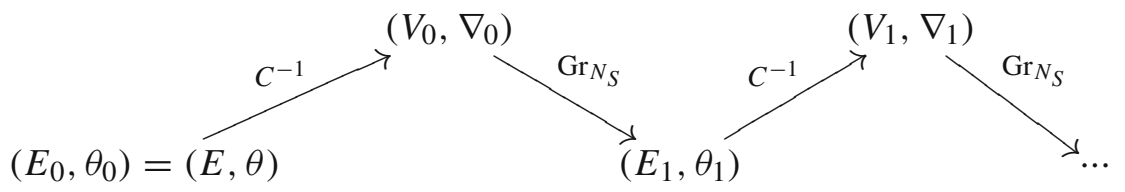

in which each $\left(V_{i}, \nabla_{i}\right)$ is slope semistable and $\left(E_{i+1}, \theta_{i+1}\right)$ is the slope semistable Higgs sheaf associated to $\left(V_{i}, \nabla_{i}\right)$ via Simpson's filtration.

As usual, in the above theorem slope semistability is defined with respect to an arbitrary fixed collection $\left(D_{1}, \ldots, D_{n-1}\right)$ of nef divisors on $X$.

\section{Bogomolov type inequalities}

In this section we use the same notation as in Sect. 2.1. Namely, $k$ is an algebraically closed field of any characteristic and $X$ is a smooth projective $k$-variety of dimension $n \geq 1$. We also fix a collection of nef divisors $\left(D_{1}, \ldots, D_{n-1}\right)$ on $X$ such that the 1 -cycle $D_{1} \ldots D_{n-1}$ is numerically nontrivial.

\subsection{Bogomolov's inequality for sheaves with generalized connections}

Let us fix a torsion free, coherent $\mathscr{O}_{X}$-module $M$ with a $k$-derivation $d_{M}$ : $\mathscr{O}_{X} \rightarrow M$. A $d_{M}$-connection (or simply an $M$-connection if $d_{M}$ is clear from the context) on a coherent $\mathscr{O}_{X}$-module $E$ is a $k$-linear map $\nabla: E \rightarrow E \otimes_{\mathscr{O}_{X}} M$ satisfying the Leibniz rule

$$
\nabla(f e)=f \nabla(e)+e \otimes d_{M}(f)
$$

for all sections $f \in \mathscr{O}_{X}$ and $e \in E$.

A useful feature of a sheaf $E$ with a $d_{M}$-connection, used below, is that if $E^{\prime} \subset E$ is an $\mathscr{O}_{X}$-submodule then the induced map $E^{\prime} \rightarrow\left(E / E^{\prime}\right) \otimes M$ is $\mathscr{O}_{X}$-linear.

From now on in this subsection we assume that $k$ has characteristic $p>0$.

Lemma 4 Let $(E, \nabla)$ be a rank $r$ slope semistable sheaf with a $d_{M}$-connection.

Let us set

$$
M_{t}=\left(F_{X}^{t}\right)^{*} M \oplus\left(F_{X}^{t-1}\right)^{*} \Omega_{X} \oplus \ldots \oplus \Omega_{X}
$$


for $t=0,1, \ldots$ If $E^{\prime} \subset\left(F_{X}^{t}\right)^{*} E$ is a saturated $\mathscr{O}_{X}$-submodule destabilizing $\left(F_{X}^{t}\right)^{*} E$ then there exists a non-zero $\mathscr{O}_{X}$-linear map $E^{\prime} \rightarrow\left(\left(F_{X}^{t}\right)^{*} E / E^{\prime}\right) \otimes M_{t}$.

Proof The proof is by induction on $t$. If $t=0$ then $E^{\prime}$ is not preserved by $\nabla$ as this would contradict semistability of $(E, \nabla)$. Then we get the required map as the one induced by $\nabla$. Now let us take $t \geq 1$ and assume the assertion holds for $\mathscr{O}_{X}$-submodules of the $(t-1)$-th Frobenius pullback of $E$. If $E^{\prime} \subset\left(F_{X}^{t}\right)^{*} E$ is not preserved by the canonical connection $\nabla_{\text {can }}$ on $\left(F_{X}\right)^{*}\left(\left(F_{X}^{t-1}\right)^{*} E\right)$ then $\nabla_{\text {can }}$ induces a non-zero $\mathscr{O}_{X}$-linear map $E^{\prime} \rightarrow\left(\left(F_{X}^{t}\right)^{*} E / E^{\prime}\right) \otimes \Omega_{X}$ and we obtain the required map by embedding $\Omega_{X}$ into $M_{t}$. Otherwise, $E^{\prime}$ is preserved by $\nabla_{\text {can }}$ and by Cartier's descent there exists an inclusion $E^{\prime \prime} \subset\left(F_{X}^{t-1}\right)^{*} E$ whose Frobenius pull back is $E^{\prime} \subset\left(F_{X}^{t}\right)^{*} E$. Since $E^{\prime \prime}$ destabilizes $\left(F_{X}^{t-1}\right)^{*} E$, by the induction assumption there exists a non-zero $\mathscr{O}_{X}$-linear map $E^{\prime \prime} \rightarrow\left(\left(F_{X}^{t-1}\right)^{*} E / E^{\prime \prime}\right) \otimes M_{t-1}$. Pulling back this map by $F_{X}$ and setting 0 on the last component, we get the required map.

Lemma 5 Let $(E, \nabla)$ be a rank $r$ torsion free sheaf with a $d_{M}$-connection. Let us set

$$
\gamma(M):=\max \left(L_{\max }(M), \frac{L_{\max }\left(\Omega_{X}\right)}{p}, 0\right) .
$$

If the collection $\left(D_{1}, \ldots, D_{n-1}\right)$ consists of ample divisors then

$$
\max \left(L_{\max }(E)-\mu_{\max }(E), \mu_{\min }(E)-L_{\min }(E)\right) \leq(r-1) \gamma(M) .
$$

Moreover, if $(E, \nabla)$ is slope semistable then

$$
L_{\max }(E)-L_{\min }(E) \leq(r-1) \gamma(M) .
$$

Proof Let us assume that $(E, \nabla)$ is semistable. By [21, Theorem 2.7] we can find $m$ such that quotients of the Harder-Narasimhan filtration for $\left(F_{X}^{m}\right)^{*} E,\left(F_{X}^{m}\right)^{*} M$ and $\left(F_{X}^{m}\right)^{*} \Omega_{X}$ are strongly semistable. Let $0=E_{0 t} \subset$ $E_{1 t} \subset \cdots \subset E_{s t}=\left(F_{X}^{t}\right)^{*} E$ be the Harder-Narasimhan filtration of $\left(F_{X}^{t}\right)^{*} E$ for $t \geq m$. Let $\mu_{i t}$ be the corresponding slopes of quotients of this filtration. By Lemma 4 there exists a non-zero $\mathscr{O}_{X}$-linear map

$$
E_{i t} \rightarrow\left(\left(F_{X}^{t}\right)^{*} E / E_{i t}\right) \otimes M_{t} .
$$

Let us choose an ample divisor $A$ such that $T_{X}(A)$ is globally generated. Then for some $N$ we have an inclusion $\Omega_{X} \subset \mathscr{O}_{X}(A)^{N}$ which gives a similar inclusion after pulling-back by the Frobenius morphisms. Hence, using 
the fact that tensor product of strongly semistable sheaves is strongly semistable (see [32, Theorem 3.23] and [22, Corollary A.3.1]), for any $t \geq m$ we have

$$
\begin{gathered}
\mu_{\min }\left(E_{i t}\right) \leq \mu_{\max }\left(\left(F_{X}^{t}\right)^{*} E / E_{i t}\right)+\max \left(\mu_{\max }\left(\left(F_{X}^{t}\right)^{*} M\right), p^{m} A D_{1} \ldots D_{t-1},\right. \\
\left.\left\{\mu_{\max }\left(\left(F_{X}^{j}\right)^{*} \Omega_{X}\right): m \leq j \leq t-1\right\}\right) .
\end{gathered}
$$

After dividing by $p^{t-m}$ this gives

$\mu_{i m} \leq \mu_{(i+1) m}+\max \left(p^{m} L_{\max }(M), p^{m-2 t} A D_{1} \ldots D_{n-1}, p^{m-1}\left[L_{\max }\left(\Omega_{X}\right)\right]_{+}\right)$.

Summing all these inequalities for $i=1, \ldots, s-1$ we get

$$
\begin{aligned}
& \mu_{1 m} \leq \mu_{s m}+(s-1) \max \left(p^{m} L_{\max }(M), p^{m-2 t} A D_{1} \ldots D_{n-1},\right. \\
& \left.p^{m-1}\left[L_{\max }\left(\Omega_{X}\right)\right]_{+}\right) .
\end{aligned}
$$

But by definition we have $\mu_{1 m}=p^{m} L_{\max }(E)$ and $\mu_{s m}=p^{m} L_{\min }(E)$ so dividing by $p^{m}$ and passing with $t$ to infinity gives the second inequality.

In particular, we proved that if $(E, \nabla)$ is semistable then

$$
\max \left(L_{\max }(E)-\mu(E), \mu(E)-L_{\min }(E)\right) \leq(r-1) \gamma(M)
$$

In general, applying this inequality to the quotients of the Harder-Narasimhan filtration of $(E, \nabla)$, we obtain the first inequality.

Theorem 6 Let $(E, \nabla)$ be a rank $r$ torsion free sheaf with a $d_{M}$-connection. Assume that the collection $\left(D_{1}, \ldots, D_{n-1}\right)$ consists of ample divisors. If $(E, \nabla)$ is slope $\left(D_{1}, \ldots, D_{n-1}\right)$-semistable then

$$
D_{1}^{2} D_{2} \ldots D_{n-1} \cdot \Delta(E) D_{2} \ldots D_{n-1}+r^{2}(r-1)^{2} \gamma(M)^{2} \geq 0
$$

In particular, if we have slope semistable $(E, \theta) \in \operatorname{HIG}(X / k)$ or $(E, \nabla) \in$ $\operatorname{MIC}(X / k)$ then

$$
D_{1}^{2} D_{2} \ldots D_{n-1} \cdot \Delta(E) D_{2} \ldots D_{n-1}+r^{2}(r-1)^{2}\left[L_{\max }\left(\Omega_{X}\right)\right]_{+}^{2} \geq 0 \text {. }
$$

Proof If $E$ is any torsion free sheaf then by [21, Theorem 5.1]

$$
\begin{aligned}
& D_{1}^{2} D_{2} \ldots D_{n-1} \cdot \Delta(E) D_{2} \ldots D_{n-1}+r^{2}\left(L_{\max }(E)-\mu(E)\right)(\mu(E) \\
& \left.\quad-L_{\min }(E)\right) \geq 0 .
\end{aligned}
$$

Therefore the theorem follows immediately from Lemma 5. 
The above theorem plays an important role in establishing Bogomolov type inequalities for semistable Higgs sheaves.

\subsection{Bogomolov's inequality for Higgs sheaves in characteristic zero}

The following Bogomolov's inequality for Higgs bundles was proven in [35, Proposition 3.4] using analytic methods. We give the first algebraic proof of this fact. For simplicity we deal only with one ample polarization although the proof works also for collections of ample polarizations.

Theorem 7 Let $k$ be a field of characteristic 0 . Let $H$ be an ample divisor on $X$. Then for any slope $H$-semistable Higgs sheaf $(E, \theta)$ we have

$$
\Delta(E) H^{n-2} \geq 0 .
$$

Proof Deforming $(E, \theta)$ to a system of Hodge sheaves (see Lemma 1) we can assume that $(E, \theta)$ is nilpotent.

Now we use a standard spreading out technique, which we recall for the convenience of the reader (cf. proof of [6, Corollaire 2.7]). There exists a subring $R \subset k$, finitely generated over $\mathbb{Z}$ and a smooth projective scheme $\mathscr{X} \rightarrow S=\operatorname{Spec} R$ such that $X=\mathscr{X} \otimes_{R} k$. We can also assume that $H$ and $(E, \theta)$ are defined over $R$ and on $\mathscr{X}$ there exists a relatively ample divisor $\mathscr{H}$ extending $H$ and an $S$-flat family of Higgs sheaves $(\mathscr{E}, \tilde{\theta})$ extending $(E, \theta)$. Further shrinking $S$, by openness of semistability we can assume that $\left(\mathscr{S}_{S}, \tilde{\theta}_{S}\right)$ is slope $\mathscr{H}_{S}$-semistable for every $s \in S$. For any closed point of $S \otimes_{\mathbb{Z}} \mathbb{Q}$ its schematic closure $T$ in $S$ is generically étale over Spec $\mathbb{Z}$. Let us choose a closed point $s \in T$ such that $T$ is étale at $s$ over $\mathbb{Z}$ and the characteristic of the residue field $k(s)$ is $\geq r=\operatorname{rk} E$. Then the scheme $\mathscr{X} \otimes_{R}$ $\left(\mathscr{O}_{\mathscr{X}, s} / m_{s}^{2}\right)$ is a lifting of $\mathscr{X}_{s}$ modulo $p^{2}$. So by Corollary 1 we can associate to $\left(\mathscr{E}_{s}, \tilde{\theta}_{s}\right)$ a slope $\mathscr{H}_{S}$-semistable sheaf with integrable connection $\left(V_{s}, \nabla_{s}\right)$. By Lemma 2 we have $\Delta\left(V_{s}\right) \mathscr{H}_{s}^{n-2}=p^{2} \Delta\left(\mathscr{E}_{s}\right) \mathscr{H}_{s}^{n-2}$. By Theorem 6 we have

$$
\mathscr{H}_{s}^{n} \cdot \Delta\left(V_{s}\right) \mathscr{H}_{s}^{n-2}+r^{2}(r-1)^{2}\left[L_{\max }\left(\Omega_{\mathscr{X}_{\bar{s}} / k(\bar{s})}\right)\right]_{+}^{2} \geq 0,
$$

where $\bar{s}$ is the geometric point lying over $s$. But for some $a \geq 0$ a suitably twisted relative tangent bundle $T_{\mathscr{X} / S}(a \mathscr{H})$ is relatively globally generated, so we can embed $\Omega_{\mathscr{X}_{\bar{s}} / k(\bar{s})}$ into a direct sum of some copies of $\mathscr{O}_{\mathscr{X}_{\bar{s}}}\left(a \mathscr{H}_{\bar{s}}\right)$. This shows that we have $L_{\max }\left(\Omega_{\mathscr{X}_{\bar{s}} / k(\bar{s})}\right) \leq a H^{n}$, where $a$ is independent of the choice of $s$. So the above inequality implies that

$$
p^{2} H^{n} \cdot \Delta(E) H^{n-2}+r^{2}(r-1)^{2}\left(a H^{n}\right)^{2} \geq 0 .
$$


Therefore dividing by $p^{2}$ and taking sufficiently large $p$ we get $\Delta(E) H^{n-2} \geq$ 0 .

Remark 1 The above inequality works also for collections of nef divisors $\left(D_{1}, \ldots, D_{n-1}\right)$ such that the 1 -cycle $D_{1} \ldots D_{n-1}$ is numerically nontrivial. However, proof of this fact requires different techniques (cf. proof of Theorem 8).

Remark 2 Note that Theorem 7 follows from Theorem 8 by the spreading out technique. We include another proof as it is much simpler and it motivates the more complicated proof of Theorem 8.

3.3 Bogomolov's inequality for Higgs sheaves in positive characteristic

Assume that $k$ has characteristic $p>0$ and that $X$ can be lifted to $W_{2}(k)$.

Theorem 8 For any slope $\left(D_{1}, \ldots, D_{n-1}\right)$-semistable Higgs sheaf $(E, \theta)$ of rank $r \leq p$ we have

$$
\Delta(E) D_{2} \ldots D_{n-1} \geq 0
$$

Proof By Lemma 1 we can deform $(E, \theta)$ to a system of Hodge sheaves preserving semistability and Chern classes. So we can assume that $(E, \theta)$ is nilpotent of level less than $r \leq p$. In this case we can consider the canonical Higgs-de-Rham sequence of $(E, \theta)$ provided by Theorem 5. By Lemma 2 we know that $c_{j}\left(V_{i}\right)=p^{j} c_{j}\left(E_{i}\right)$ for $i=0, \ldots$ On the other hand, it is clear that $c_{j}\left(E_{i+1}\right)=c_{j}\left(V_{i}\right)$, so we have

$$
\Delta\left(E_{i}\right) D_{2} \ldots D_{n-1}=p^{2 i} \Delta(E) D_{2} \ldots D_{n-1} .
$$

Let us set $d=D_{1}^{2} D_{2} \ldots D_{n-1} \geq 0$.

First, let us consider the case in which all the divisors $D_{1}, \ldots, D_{n-1}$ are ample. Then by Theorem 6 we have

$$
d \cdot \Delta\left(E_{i}\right) D_{2} \ldots D_{n-1}+r^{2}(r-1)^{2}\left[L_{\max }\left(\Omega_{X}\right)\right]_{+}^{2} \geq 0 .
$$




$$
\Delta(E) D_{2} \ldots D_{n-1} \geq-\frac{r^{2}(r-1)^{2}\left[L_{\max }\left(\Omega_{X}\right)\right]_{+}^{2}}{d p^{2 i}}
$$

and we get the required inequality passing with $i$ to infinity. Note that this implies that if $D_{1}, \ldots, D_{n-1}$ are ample then

$$
\begin{aligned}
& D_{1}^{2} D_{2} \ldots D_{n-1} \cdot \Delta(E) D_{2} \ldots D_{n-1}+r^{2}\left(\mu_{\max }(E, \theta)\right. \\
& \quad-\mu(E))\left(\mu(E)-\mu_{\min }(E, \theta)\right) \geq 0
\end{aligned}
$$

(see Corollary 3).

Now let us consider the general case. The strategy is the same as that in proof of [21, 3.6 and 3.8] and we just sketch the arguments. First, let us assume that $d>0$. In this case we fix an ample divisor $H$ and set $H_{i}(t)=D_{i}+t H$. Then the Harder-Narasimhan filtration of $(E, \theta)$ with respect to $\left(H_{1}(t), \ldots, H_{n-1}(t)\right)$ is independent of $t$ for small positive $t$ (this is a not-trivial assertion; see [21, 3.6] for proof in the case of usual sheaves; the proof in our case is the same).

Let $0=F_{0} \subset F_{1} \subset \cdots \subset F_{m}=(E, \theta)$ be the corresponding filtration. Since $(E, \theta)$ is slope $\left(D_{1}, \ldots, D_{n-1}\right)$-semistable, we have

$\lim _{t \rightarrow 0} \mu_{\max , H_{1}(t), \ldots, H_{n-1}(t)}(E, \theta)=\lim _{t \rightarrow 0} \mu_{H_{1}(t), \ldots, H_{n-1}(t)}\left(F_{1}\right)=\mu_{D_{1}, \ldots, D_{n-1}}(E)$

and

$$
\lim _{t \rightarrow 0} \mu_{\min , H_{1}(t), \ldots, H_{n-1}(t)}(E, \theta)=\mu_{D_{1}, \ldots, D_{n-1}}(E) .
$$

But we know that

$$
\begin{aligned}
& H_{1}(t)^{2} H_{2}(t) \ldots H_{n-1}(t) \cdot \Delta(E) H_{2}(t) \ldots H_{n-1}(t) \\
& \quad+r^{2}\left(\mu_{\max , H_{1}(t), \ldots, H_{n-1}(t)}(E, \theta)-\mu_{H_{1}(t), \ldots, H_{n-1}(t)}(E)\right) \cdot\left(\mu_{H_{1}(t), \ldots, H_{n-1}(t)}(E)\right. \\
& \left.\quad-\mu_{\min , H_{1}(t), \ldots, H_{n-1}(t)}(E, \theta)\right) \geq 0,
\end{aligned}
$$

so passing with $t$ to 0 yields the required inequality.

Now consider the remaining case $d=0$ and assume that $\Delta(E) D_{2} \ldots D_{n-1}$ $<0$. Let us choose an ample divisor $H_{1}$ such that $H_{1}^{2} D_{2} \ldots D_{n-1}>0$. Then $(E, \theta)$ is not slope $\left(H_{1}, D_{2}, \ldots, D_{n-1}\right)$-semistable and as in $[21,3.7]$ (see also [12, Theorem 7.3.3]) one can show that there exists a saturated Higgs subsheaf $\left(E^{\prime}, \theta^{\prime}\right) \subset(E, \theta)$ such that $\left(c_{1}\left(E^{\prime}\right) / r^{\prime}-c_{1}(E) / r\right) D_{1}^{\prime} D_{2} \ldots D_{n-1}>0$ for every nef divisor $D_{1}^{\prime}$ such that $D_{1}^{\prime} D_{2} \ldots D_{n-1}$ is numerically nontrivial. In particular, for $D_{1}^{\prime}=D_{1}$ we get a contradiction with our assumption on $\left(D_{1}, \ldots, D_{n-1}\right)$ semistability of $(E, \theta)$.

The following corollary is a generalization of Theorem 1: 
Corollary 2 For any $\left(D_{1}, \ldots, D_{n-1}\right)$-semistable sheaf $E$ of rank $r \leq p$ we have

$$
\Delta(E) D_{2} \ldots D_{n-1} \geq 0
$$

The above corollary was known only in the surface case for rank 2 vector bundles in characteristic 2 (see [34, Corollary 11]).

Corollary 3 If $(E, \theta)$ is a torsion free Higgs sheaf of rank $r \leq p$ then

$$
\begin{aligned}
& D_{1}^{2} D_{2} \ldots D_{n-1} \cdot \Delta(E) D_{2} \ldots D_{n-1}+r^{2}\left(\mu_{\max }(E, \theta)\right. \\
& \quad-\mu(E))\left(\mu(E)-\mu_{\min }(E, \theta)\right) \geq 0 .
\end{aligned}
$$

Proof Let $0=F_{0} \subset F_{1} \subset \cdots \subset F_{m}=(E, \theta)$ be the Harder-Narasimhan filtration (in the category of Higgs sheaves). Set $F^{i}=F_{i} / F_{i-1}, r_{i}=\operatorname{rk} F^{i}, \mu_{i}=$ $\mu\left(F^{i}\right)$. We can assume that $d=D_{1}^{2} D_{2} \ldots D_{n-1}>0$. Then by the Hodge index theorem we have

$$
\begin{aligned}
\frac{\Delta(E) D_{2} \ldots D_{n-1}}{r}= & \sum \frac{\Delta\left(F^{i}\right) D_{2} \ldots D_{n-1}}{r_{i}} \\
& -\frac{1}{r} \sum_{i<j} r_{i} r_{j}\left(\frac{c_{1} F^{i}}{r_{i}}-\frac{c_{1} F^{j}}{r_{j}}\right)^{2} D_{2} \ldots D_{n-1} \\
\geq & \sum \frac{\Delta\left(F^{i}\right) D_{2} \ldots D_{n-1}}{r_{i}}-\frac{1}{r d} \sum_{i<j} r_{i} r_{j}\left(\mu_{i}-\mu_{j}\right)^{2} .
\end{aligned}
$$

Since $\Delta\left(F^{i}\right) D_{2} \ldots D_{n-1} \geq 0$ by Theorem 8 , the required inequality follows from the following easy inequality (see [21, Lemma 1.4])

$$
\sum_{i<j} r_{i} r_{j}\left(\mu_{i}-\mu_{j}\right)^{2} \leq r^{2}\left(\mu_{1}-\mu(E)\left(\mu(E)-\mu_{m}\right) .\right.
$$

\section{Restriction theorems}

In this section we assume that $k$ has characteristic $p>0$ and that $X$ can be lifted to $W_{2}(k)$. We recall how Bogomolov's inequality implies effective restriction theorems. Originally, this method was invented by F. Bogomolov with further improvements by the author (see [21, Theorem 5.2]). We give all the details as we feel that this approach is not as known as it should be and some people still reprove much weaker restriction theorems in a more complicated way. 
Note that in the characteristic zero case a non-effective version of restriction theorem (the so called Mehta-Ramanathan restriction theorem) together with Bogomolov's inequality in the surface case implies Bogomolov's inequality in higher dimensions. This is a classical approach and it was also used by Mochizuki in $[28,29]$ to deduce Bogomolov's inequality for semistable (logarithmic) Higgs sheaves in higher dimensions. Our approach is exactly opposite: first we prove Bogomolov's inequality in all dimensions and then we deduce (strong) restriction theorems.

\subsection{Restriction theorem for sheaves with operators}

First, we repeat arguments from proof of [21, Theorem 5.2] to prove a strong restriction theorem for sheaves with operators:

Theorem 9 Let $(E, \theta)$ be a torsion free Higgs sheaf of rank $r \leq p$. Assume that $(E, \theta)$ is slope $\left(D_{1}, \ldots, D_{n-1}\right)$-stable and $d=D_{1}^{2} D_{2} \ldots D_{n-1}>0$. Let $D \in\left|m D_{1}\right|$ be a normal divisor such that $\left.E\right|_{D}$ has no torsion. If

$$
m>\frac{r-1}{r} \Delta(E) D_{2} \ldots D_{n-1}+\frac{1}{d r(r-1)}
$$

then $\left(\left.E\right|_{D},\left.\theta\right|_{D}\right)$ is a slope $\left(\left.D_{2}\right|_{D}, \ldots,\left.D_{n-1}\right|_{D}\right)$-stable sheaf with an $\left.\Omega_{X}\right|_{D^{-}}$ valued operator.

Proof Let us assume that $\left(\left.E\right|_{D},\left.\theta\right|_{D}\right)$ is not slope $\left(\left.D_{2}\right|_{D}, \ldots,\left.D_{n-1}\right|_{D}\right)$-stable. Let $\left(T, \theta_{T}:\left.T \rightarrow T \otimes \Omega_{X}\right|_{D}\right)$ be the minimal destabilizing quotient of $\left(\left.E\right|_{D},\left.\theta\right|_{D}\right)$. Let us set $\rho=\operatorname{rk} T$ and let $E^{\prime}$ be the kernel of the composition $\left.E \rightarrow E\right|_{D} \rightarrow T$. Note that $E^{\prime}$ has a natural structure of a Higgs subsheaf of $(E, \theta)$ with Higgs field $\theta^{\prime}=\left.\theta\right|_{E^{\prime}}$. Since $\left(T, \theta_{T}\right)$ destabilizes $\left(\left.E\right|_{D},\left.\theta\right|_{D}\right)$ we have

$$
\begin{aligned}
& \Delta\left(E^{\prime}\right) D_{2} \ldots D_{n-1}=\Delta(E) D_{2} \ldots D_{n-1}-\rho(r-\rho) D^{2} D_{2} \ldots D_{n-1} \\
& \quad+2\left(r c_{1}(T)-\rho c_{1}\left(\left.E\right|_{D}\right)\right) D_{2} \ldots D_{n-1} \leq \Delta(E) D_{2} \ldots D_{n-1}-\rho(r-\rho) m^{2} d .
\end{aligned}
$$

Since $\left(E^{\prime}, \theta^{\prime}\right) \subset(E, \theta)$ and $(E, \theta)$ is slope $\left(D_{1}, \ldots, D_{n-1}\right)$-stable we have

$$
\begin{aligned}
\mu_{\max }\left(E^{\prime}, \theta^{\prime}\right)-\mu\left(E^{\prime}\right) & =\frac{\rho}{r} D D_{1} \ldots D_{n-1}+\mu_{\max }\left(E^{\prime}, \theta^{\prime}\right)-\mu(E) \\
& \leq \frac{\rho}{r} m d-\frac{1}{r(r-1)} .
\end{aligned}
$$


Similarly, since $(E(-D), \theta(-D)) \subset\left(E^{\prime}, \theta^{\prime}\right)$ we have

$$
\begin{aligned}
\mu\left(E^{\prime}\right)-\mu_{\min }\left(E^{\prime}, \theta^{\prime}\right) & =\frac{r-\rho}{r} D D_{1} \ldots D_{n-1}+\mu(E(-D))-\mu_{\min }\left(E^{\prime}, \theta^{\prime}\right) \\
& \leq \frac{r-\rho}{r} m d-\frac{1}{r(r-1)} .
\end{aligned}
$$

Hence by Corollary 3 we obtain

$$
\begin{aligned}
0 \leq & d \cdot \Delta\left(E^{\prime}\right) D_{2} \ldots D_{n-1}+r^{2}\left(\mu_{\max }\left(E^{\prime}, \theta^{\prime}\right)-\mu\left(E^{\prime}\right)\right)\left(\mu\left(E^{\prime}\right)-\mu_{\min }\left(E^{\prime}, \theta^{\prime}\right)\right) \\
\leq & d \cdot \Delta(E) D_{2} \ldots D_{n-1}-\rho(r-\rho) m^{2} d^{2}+\left(\rho m d-\frac{1}{r-1}\right) \\
& \times\left((r-\rho) m d-\frac{1}{r-1}\right) .
\end{aligned}
$$

Therefore

$$
m \frac{d r}{r-1} \leq d \cdot \Delta(E) D_{2} \ldots D_{n-1}+\frac{1}{(r-1)^{2}}
$$

which contradicts our assumption on $m$.

\subsection{Restriction theorem for Higgs sheaves}

Note that up to now even in characteristic zero there were no effective vanishing theorems for Higgs semistability ${ }^{1}$. Here we use Simpson's arguments from proof of [36, Lemma 3.7] to prove the first such result:

Theorem 10 Let $(E, \theta)$ be a torsion free Higgs sheaf of rank $r \leq p$. Let $m_{0}$ be a non-negative integer such that $T_{X}\left(m_{0} D_{1}\right)$ is globally generated. Assume that $(E, \theta)$ is slope $\left(D_{1}, \ldots, D_{n-1}\right)$-stable and $d=D_{1}^{2} D_{2} \ldots D_{n-1}>0$. Let $D \in\left|m D_{1}\right|$ be a normal divisor such that $\left.E\right|_{D}$ has no torsion. Let us take an integer

$$
m>\frac{r-1}{r} \Delta(E) D_{2} \ldots D_{n-1}+\frac{1}{d r(r-1)} .
$$

\footnotetext{
1 The only published result in this direction, due to Biswas and Dey (in Bull. Sci. Math. 135 (2011), 178-186) is incorrect. The proof does not show any restriction theorem for Higgs sheaves but only a restriction theorem for sheaves with operators, which is a weak form of Theorem 9 in the characteristic zero case.
} 
Moreover, if $r>2$ let us also assume that $m>2(r-1)^{2} m_{0}$. Then the Higgs sheaf $\left(\left.E\right|_{D}, \theta_{D}\right)$, induced from $\left(\left.E\right|_{D},\left.\theta\right|_{D}\right)$ via composition $\left.E\right|_{D} \rightarrow$ $\left.\left.E\right|_{D} \otimes \Omega_{X}\right|_{D} \rightarrow E \otimes \Omega_{D}$, is slope $\left(\left.D_{2}\right|_{D}, \ldots,\left.D_{n-1}\right|_{D}\right)$-stable.

Proof Let us assume that $\left(\left.E\right|_{D}, \theta_{D}\right)$ is not slope $\left(\left.D_{2}\right|_{D}, \ldots,\left.D_{n-1}\right|_{D}\right)$-stable and let $\left(E^{\prime}, \theta^{\prime}\right) \subset\left(\left.E\right|_{D}, \theta_{D}\right)$ be the maximal destabilizing Higgs subsheaf. By Theorem $9\left(\left.E\right|_{D},\left.\theta\right|_{D}\right)$ is slope $\left(\left.D_{2}\right|_{D}, \ldots,\left.D_{n-1}\right|_{D}\right)$-stable. Therefore $E^{\prime}$ is not preserved by $\left.\theta\right|_{D}$ and we have a non-zero map $\left.E^{\prime} \rightarrow E^{\prime \prime} \otimes \Omega_{X}\right|_{D}$, where $E^{\prime \prime}=E / E^{\prime}$. This induces a non-zero map $\varphi: E^{\prime} \rightarrow E^{\prime \prime}(-D)$. In particular, if $r=2$ then $E^{\prime}$ and $E^{\prime \prime}$ have rank one and we have a contradiction with $\mu\left(E^{\prime}\right) \geq \mu\left(E^{\prime \prime}\right)>\mu\left(E^{\prime \prime}(-D)\right)$.

If $r \geq 3$ we need more subtle arguments. Let $G=\operatorname{ker} \varphi$ and let $M$ be the preimage of $\left(E^{\prime} / G\right)(D) \subset E^{\prime \prime}$ under the canonical projection $\left.E\right|_{D} \rightarrow E^{\prime \prime}$. Then

$\operatorname{deg} M=\operatorname{deg} E^{\prime}+\operatorname{deg}\left(E^{\prime} / G\right)(D)=2 \operatorname{deg} E^{\prime}-\operatorname{deg} G+\operatorname{rk}\left(E^{\prime} / G\right) \operatorname{deg} \mathscr{O}_{D}(D)$.

Therefore

$$
\operatorname{deg} M+\operatorname{deg} G=2 \operatorname{deg} E^{\prime}+\operatorname{rk}\left(E^{\prime} / G\right) \operatorname{deg} \mathscr{O}_{D}(D) \geq 2 r^{\prime} \mu(E)+m^{2} d .
$$

But $M$ and $G$ are subsheaves of $\left.E\right|_{D}$, so

$$
\operatorname{deg} M+\operatorname{deg} G \leq(\operatorname{rk} M+\operatorname{rk} G) \mu_{\max }\left(\left.E\right|_{D}\right)=2 r^{\prime} \mu_{\max }\left(\left.E\right|_{D}\right) .
$$

Since $\left(\left.E\right|_{D},\left.\theta\right|_{D}\right)$ is slope $\left(\left.D_{2}\right|_{D}, \ldots,\left.D_{n-1}\right|_{D}\right)$-stable, by Lemma 5 we have

$$
\mu_{\max }\left(\left.E\right|_{D}\right)-\mu_{\min }\left(\left.E\right|_{D}\right) \leq(r-1)\left[L_{\max }\left(\left.\Omega_{X}\right|_{D}\right)\right]_{+} .
$$

But $T_{X}\left(m_{0} D_{1}\right)$ is globally generated, so $\left.\Omega_{X}\right|_{D} \subset \mathscr{O}_{D}\left(m_{0} D_{1}\right)^{\oplus N}$ for some $N$. This shows that $L_{\max }\left(\left.\Omega_{X}\right|_{D}\right) \leq m_{0} m d$ and hence

$$
2 r^{\prime} \mu(E)+m^{2} d \leq \operatorname{deg} M+\operatorname{deg} G \leq 2 r^{\prime}\left(\mu(E)+(r-1) m_{0} m d\right) .
$$

This contradicts our assumption that $m>2(r-1)^{2} m_{0}$.

Let us remark that in both Theorems 9 and 10 we do not assume that the divisor $D$, to which we restrict the Higgs bundle, lifts to $W_{2}(k)$. Moreover, both theorems (and their proofs) work also in the characteristic zero case.

\section{Semistable Higgs sheaves with vanishing Chern classes}

We keep the same notation and assumptions as in the previous section (unless otherwise explicitly stated). 
In this section we study semistable Higgs sheaves with vanishing Chern classes. In the characteristic zero case such sheaves correspond to representations of the topological fundamental group. Here they also exhibit analogous interesting properties. For example, we prove that they are locally free and satisfy a strong restriction theorem. It is also interesting to note that one can define on them certain discrete dynamical systems (even in characteristic zero). This fact seems to be unnoticed in the characteristic zero case and the corresponding systems were not yet studied. In positive characteristic we make the first steps in this direction.

\subsection{Local freeness}

In this subsection we prove that semistable Higgs sheaves with vanishing Chern classes are locally free. This is a positive characteristic version of [36, Theorem 2] (see also [23, Theorem 4.1] for another version for strongly semistable sheaves). This fact can be also considered as a vast generalization of [18, Corollary 3.11], which was proven by a completely different method using Faltings' [9, Theorem 2.1]. Actually, [18, Corollary 3.11] implies that preperiodic Higgs sheaves (see Sect. 5.3) are locally free. Indeed, by [18, Corollary 3.11] there exists $i$ such that $E_{i}$ in the Higgs-de Rham sequence of preperiodic $(E, \theta)$ is locally free. So by Lemma $3 E$ is locally free. In particular, Theorem 5 implies Theorem 11 for an algebraic closure of a finite field.

This theorem has many interesting applications. In particular, it implies that moduli spaces of semistable Higgs bundles with vanishing Chern classes are projective. This is important as it is much easier to deal with locally free sheaves than torsion free ones, e.g., if one needs to study moduli spaces of principal Higgs bundles. Another corollary says that the category of slope semistable Higgs bundles of rank $r \leq p$ with vanishing Chern classes behaves similarly to the category of representations of the fundamental group (cf. Corollary 5).

Theorem 11 Let $H$ be an ample divisor on $X$ and let $(E, \theta)$ be a rank $r \leq p$ slope $H$-semistable Higgs sheaf with $\mathrm{ch}_{1}(E) H^{n-1}=0$ and $\mathrm{ch}_{2}(E) H^{n-2}=0$. Assume that either $E$ is reflexive or the normalized Hilbert polynomial of $E$ is the same as that of $\mathscr{O}_{X}$. Then $E$ has a filtration whose quotients are locally free slope $H$-stable Higgs sheaves with vanishing Chern classes.

Note: We say that $E$ has vanishing Chern classes if the Chern classes $c_{i}(E)$ vanish in $H_{\mathrm{et}}^{2 i}\left(X, \mathbb{Q}_{l}(i)\right)$ for $i>0$ and any $l \neq p$. It also implies that numerically Chern classes are trivial (in particular, $c_{i}(E) H^{n-i}=0$ ).

Proof In the curve case the theorem follows from existence of a Jordan-Hölder filtration. In general, the proof is by induction on the dimension $n$ starting with $n=2$. 
If $X$ is a surface then the Higgs field $\theta$ extends to a Higgs field $\tilde{\theta}$ on the reflexivization $E^{* *}$ of $E$. Note that $E^{* *}$ is locally free and $\left(E^{* *}, \tilde{\theta}\right)$ is slope $H$ semistable. Hence $\Delta\left(E^{* *}\right) \geq 0$ by Theorem 8 . By the Hodge index theorem we have

$$
0=2 r \operatorname{ch}_{2}(E)=c_{1}(E)^{2}-\Delta(E) \leq c_{1}(E)^{2} \leq \frac{\left(c_{1}(E) H\right)^{2}}{H^{2}}=0,
$$

so $c_{1}(E)^{2}=\Delta(E)=0$. But $\Delta\left(E^{* *}\right) \leq \Delta(E)$ so also $\Delta\left(E^{* *}\right)=0$. Since $E^{* *} / E$ is supported on a finite set of points and its second Chern class vanishes, we must have $E=E^{* *}$. Hence the required assertion can be obtained by taking a Jordan-Hölder filtration of $(E, \theta)$.

Now fix $n \geq 3$ and assume that the theorem holds for varieties of dimension less than $n$. First, let us assume that $E$ is reflexive. By the same argument as above, we see that $c_{1}(E) H^{n-1}=0$ and $\Delta(E) H^{n-2}=0$. Let us consider the canonical Higgs-de Rham sequence of $(E, \theta)$ provided by Theorem 5. By [21, Theorem 4.4] the family of Higgs sheaves $\left(E_{i}, \theta_{i}\right)$ is bounded. Therefore there are only finitely many possibilities for Chern classes $c_{j}\left(E_{i}\right)=p^{i j} c_{j}(E)$. This implies that the Chern classes of $E$ must vanish. In particular, for any smooth divisor $D$ on $X$, the reduced Hilbert polynomial of $E_{D}$ is equal to the Hilbert polynomial of $\mathscr{O}_{D}$. Moreover, $E_{D}$ is torsion free (see, e.g., [12, Corollary 1.1.14]).

Let us assume that $(E, \theta)$ is slope $H$-stable. Without loss of generality we can assume that $H^{1}\left(X, \mathscr{O}_{X}(m H)\right)=0$ for $m \geq 1$ and $\mathscr{O}_{X}(H)$ lifts to a line bundle $\mathscr{O}_{\tilde{X}}(\tilde{H})$ on a lifting $\tilde{X}$ of $X$ to $W_{2}(k)$. Then a short exact sequence

$$
0 \rightarrow p \mathscr{O}_{\tilde{X}} \rightarrow \mathscr{O}_{\tilde{X}} \rightarrow \mathscr{O}_{X} \rightarrow 0
$$

implies that $H^{0}\left(\mathscr{O}_{\tilde{X}}(m \tilde{H})\right) \rightarrow H^{0}\left(\mathscr{O}_{X}(m H)\right)$ is surjective for all $m \geq 1$ (note that $p \mathscr{O}_{\tilde{X}} \simeq \mathscr{O}_{X}$ as $\mathscr{O}_{X}$-modules). By Theorem 10 the restriction $E_{D}$ is also slope $H$-stable for any smooth divisor $D \in|m H|, m \geq 1$. Since by the above $D$ can be lifted to $W_{2}(k)$, the restriction $E_{D}$ is locally free by the induction assumption. It follows that $E$ is locally free along every smooth divisor $D \in|m H|$. Since such divisors cover $X$ (when we vary $m$ ) it follows that $E$ is locally free.

The general case can be proven by induction on the rank $r$ of $E$. Let us take any saturated proper Higgs subsheaf $\left(E^{\prime}, \theta^{\prime}\right) \subset(E, \theta)$ which is slope $H$-stable with $c_{1}\left(E^{\prime}\right) H^{n-1}=0$. Let us set $\left(E^{\prime \prime}, \theta^{\prime \prime}\right)=(E, \theta) /\left(E^{\prime}, \theta^{\prime}\right)$. By the Hodge index theorem and Theorem 8 we have

$$
0=\frac{\Delta(E) H^{n-2}}{r}=\frac{\Delta\left(E^{\prime}\right) H^{n-2}}{r^{\prime}}+\frac{\Delta\left(E^{\prime \prime}\right) H^{n-2}}{r^{\prime \prime}}-\frac{r^{\prime} r^{\prime \prime}}{r}\left(\frac{c_{1} E^{\prime}}{r^{\prime}}-\frac{c_{1} E^{\prime \prime}}{r^{\prime \prime}}\right)^{2} H^{n-2}
$$




$$
\geq \frac{\Delta\left(E^{\prime}\right) H^{n-2}}{r^{\prime}}+\frac{\Delta\left(E^{\prime \prime}\right) H^{n-2}}{r^{\prime \prime}} \geq 0 .
$$

So $\Delta\left(E^{\prime}\right) H^{n-2}=0$ and $\Delta\left(E^{\prime \prime}\right) H^{n-2}=0$ and hence

$$
\begin{aligned}
0 & =\operatorname{ch}_{2}(E) H^{n-2}=\operatorname{ch}_{2}\left(E^{\prime}\right) H^{n-2}+\operatorname{ch}_{2}\left(E^{\prime \prime}\right) H^{n-2} \\
& =c_{1}\left(E^{\prime}\right)^{2} H^{n-2}+c_{1}\left(E^{\prime \prime}\right)^{2} H^{n-2} .
\end{aligned}
$$

By the Hodge index theorem

$$
c_{1}\left(E^{\prime}\right)^{2} H^{n-2} \leq \frac{\left(c_{1}\left(E^{\prime}\right) H^{n-1}\right)^{2}}{H^{n}}=0
$$

and

$$
c_{1}\left(E^{\prime \prime}\right)^{2} H^{n-2} \leq \frac{\left(c_{1}\left(E^{\prime \prime}\right) H^{n-1}\right)^{2}}{H^{n}}=0 .
$$

It follows that $\operatorname{ch}_{1}\left(E^{\prime}\right) H^{n-1}=0$ and $\operatorname{ch}_{2}\left(E^{\prime}\right) H^{n-2}=0$. Since $E^{\prime}$ is reflexive, by the induction assumption it is locally free with vanishing Chern classes. But then $E^{\prime \prime}$ also has vanishing Chern classes. Therefore the reduced Hilbert polynomial of $E^{\prime \prime}$ is equal to the Hilbert polynomial of $\mathscr{O}_{X}$. Since $\left(E^{\prime \prime}, \theta^{\prime \prime}\right)$ is slope $H$-semistable, by the induction assumption it has a filtration whose quotients are locally free slope $H$-stable Higgs sheaves with vanishing Chern classes.

Now we assume that the reduced Hilbert polynomial of $E$ is equal to the Hilbert polynomial of $\mathscr{O}_{X}$ without assuming that $E$ is reflexive. By what we already proved the reflexivization $E^{* *}$ of $E$ is locally free with vanishing Chern classes. Therefore the reduced Hilbert polynomial of $E^{* *}$ is also equal to the Hilbert polynomial of $\mathscr{O}_{X}$. This implies that the Hilbert polynomial of the quotient $E^{* *} / E$ is trivial, which proves the required assertion.

Corollary 4 Let $H$ be an ample divisor on $X$ and let $(E, \theta)$ be a rank $r \leq p$ slope $H$-semistable Higgs sheaf with vanishing Chern classes. Then $(E, \theta)$ is locally free and Gieseker semistable (and hence also slope semistable) with respect to every ample polarization.

Proof Since by assumption the normalized Hilbert polynomial of $E$ is the same as that of $\mathscr{O}_{X}$, Theorem 11 implies that $E$ is locally free. Let us recall that the family of Higgs sheaves $\left\{\left(E_{i}, \theta_{i}\right)\right\}_{i \in \mathbb{Z}_{\geq 0}}$ in the canonical Higgs-de Rham sequence of $(E, \theta)$ is bounded (see proof of Theorem 11).

Assume that $(E, \theta)$ is not slope $A$-semistable for some ample polarization $A$ and consider the maximal $A$-destabilizing Higgs subsheaf $\left(E^{\prime}, \theta^{\prime}\right) \subset(E, \theta)$. 
Then by Lemma 2 the slopes (counted with respect to $A$ ) of images of $\left(E^{\prime}, \theta^{\prime}\right)$ under iterations of $\mathrm{Gr}_{N_{S}} \circ C^{-1}$ grow to infinity. But this contradicts boundedness of the family $\left\{\left(E_{i}, \theta_{i}\right)\right\}_{i \in \mathbb{Z}_{\geq 0}}$.

Hence $(E, \theta)$ is slope $A$-semistable. By Theorem 11 it has a filtration whose quotients are locally free slope $A$-stable Higgs sheaves with vanishing Chern classes, so it is also Gieseker $A$-semistable.

Corollary 5 Let $\mathscr{C}$ be the full subcategory of the category of coherent Higgs sheaves on $X$, whose objects are slope semistable Higgs bundles of rank $r \leq$ $p$ with vanishing Chern classes. Then $\mathscr{C}$ contains all kernels, images and cokernels.

Proof Let $\left(E_{1}, \theta_{1}\right)$ and $\left(E_{2}, \theta_{2}\right)$ be two objects of $\mathscr{C}$. By Theorem 11 both $\left(E_{1}, \theta_{1}\right)$ and $\left(E_{2}, \theta_{2}\right)$ have filtrations by slope stable objects of $\mathscr{C}$. Since the only maps of Higgs sheaves between such slope stable objects are either zero or isomorphisms, it is easy to see that the kernel, image and cokernel of any morphism of Higgs sheaves $\left(E_{1}, \theta_{1}\right) \rightarrow\left(E_{2}, \theta_{2}\right)$ are objects of $\mathscr{C}$.

Note that Theorem 11 also implies [36, Theorem 2] using a standard spreading out argument (or following proof of Theorem 11). This gives the first algebraic proof of Simpson's result (original Simpson's arguments use Lefschetz's hyperplane theorem for the topological fundamental group).

Corollary 6 Let $X$ be a smooth projective variety defined over a field of characteristic zero. Let $H$ be an ample divisor on $X$ and let $(E, \theta)$ be a slope $H$-semistable Higgs sheaf with $\mathrm{ch}_{1}(E) H^{n-1}=0$ and $\operatorname{ch}_{2}(E) H^{n-2}=0$. Assume that either $E$ is reflexive or the normalized Hilbert polynomial of $E$ is the same as that of $\mathscr{O}_{X}$. Then $E$ has a filtration whose quotients are locally free slope $H$-stable Higgs sheaves with vanishing Chern classes.

\subsection{Bruzzo-Hernández Ruipérez' strong restriction theorem}

It is well known that on a smooth complex variety, a slope semistable locally free sheaf with vanishing Chern classes is numerically flat, i.e., its restriction to every curve is nef of degree 0 . A similar theorem for Higgs sheaves was proven by Bruzzo and Hernández Ruipérez in [5]. The known proofs of these theorems use the fact that a vector bundle of degree 0 on a smooth complex projective curve is semistable if and only if it is nef. This is no longer true in positive characteristic as nefness characterizes strong semistability. However, we can prove that the same restriction theorem holds also in positive characteristic. Note that this theorem immediately implies the characteristic zero version by standard reduction to positive characteristic (cf. proof of Theorem 7). 
Theorem 12 Let $E$ be a locally free $\mathscr{O}_{X}$-module of rank $r \leq p=$ char $k$ with vanishing Chern classes. Assume that a Higgs sheaf $(E, \theta)$ is slope $H$ semistable for some ample polarization $H$. Let $v: \tilde{C} \rightarrow C \subset X$ be a normalization of some projective curve. Then the induced Higgs bundle $\left(\nu^{*} E, \theta_{\tilde{C}}\right)$ is (slope) semistable.

Proof Let us consider the canonical Higgs-de Rham sequence of $(E, \theta)$ from Theorem 5. By Theorem 11 all the involved sheaves $V_{i}$ and $E_{i}$ are locally free and have vanishing Chern classes (note that we need this theorem even though we assume that $E$ is locally free with vanishing Chern classes!). Therefore by functoriality of the Cartier transform we have the induced Higgs-de Rham sequence for $\left(v^{*} E, \theta_{\tilde{C}}\right)$ :

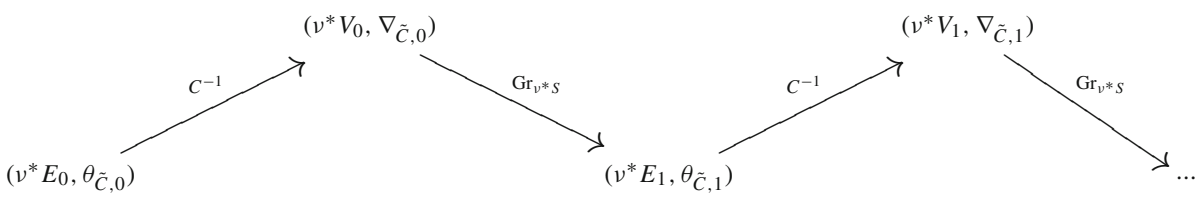

Since the Higgs sheaves $\left\{\left(E_{i}, \theta_{i}\right)\right\}_{i \geq 0}$ are all locally free with vanishing Chern classes and they are all slope $H$-semistable, they form a bounded set of sheaves. Therefore $\left\{v^{*} E_{i}\right\}_{i \geq 0}$ also forms a bounded set of sheaves on $\tilde{C}$. Assume that $\left(v^{*} E, \theta_{\tilde{C}}\right)$ is not semistable and let $\left(E^{\prime}, \theta^{\prime}\right)$ denote its maximal destabilizing Higgs subsheaf. In particular, $\mu\left(E^{\prime}\right)>\mu\left(v^{*} E\right)=0$. Then the filtration $v^{*} S$ induces on $C^{-1}\left(E^{\prime}, \theta^{\prime}\right) \subset\left(v^{*} V_{0}, \nabla_{\tilde{C}, 0}\right)$ a Griffiths transverse filtration such that the associated Higgs sheaf is a Higgs subsheaf of $\left(v^{*} E_{1}, \theta_{\tilde{C}, 1}\right)$ of slope $p \mu\left(E^{\prime}\right)$. Analogously, $v^{*} E_{i}$ contains a subsheaf of slope $p^{i} \mu\left(E^{\prime}\right)$, which is impossible for large $i$.

\subsection{Discrete dynamical systems on Higgs bundles}

Let $(E, \theta)$ be a rank $r \leq p$ torsion free Higgs sheaf with nilpotent Higgs field.

Definition 2 We say that $(E, \theta)$ is periodic if there exists some positive $i$ such that in the canonical Higgs-de Rham sequence of $(E, \theta)$ we have $\left(E_{i}, \theta_{i}\right) \simeq$ $(E, \theta)$. We say that $(E, \theta)$ is preperiodic if there exists some $i$ such that $\left(E_{i}, \theta_{i}\right)$ is periodic.

The following proposition strengthens (and corrects) [18, Theorem 2.5] and [18, Corollary 3.11]:

Proposition 1 1. If $(E, \theta)$ is preperiodic then it is locally free with vanishing Chern classes. Moreover, $(E, \theta)$ is slope semistable with respect to every ample polarization. 
2. If $k=\overline{\mathbb{F}}_{p}$ and $(E, \theta)$ is slope semistable with vanishing Chern classes then it is preperiodic.

Proof If $(E, \theta)$ is preperiodic then by Lemma 2 its Chern classes vanish. Then Theorem 11 implies that $E$ is locally free (for another argument in case $k=\overline{\mathbb{F}}_{p}$ see the beginning of Sect. 5.1). Slope semistability can be proven in the same way as Corollary 4.

The second part of the proposition follows from Theorem 5 and [18, Theorem 2.5]. For the convenience of the reader we repeat a simplified version of proof of [18, Theorem 2.5]. Let us consider the canonical Higgs-de Rham sequence of $(E, \theta)$. Note that $\mathrm{Gr}_{S} \circ C^{-1}$ does not enlarge the field of definition of $\left(E_{i}, \theta_{i}\right)$ (this requires the fact that Simpson's filtration is preserved by the action of the Galois group). So we can assume that $X$ and all the bundles $\left(E_{i}, \theta_{i}\right)$ are defined over the same finite field. But the Higgs bundles $\left\{\left(E_{i}, \theta_{i}\right)\right\}_{i \in \mathbb{Z}_{>0}}$ are slope semistable with vanishing Chern classes so by Lemma 5 and [21, Theorem 4.4] they form a bounded family. Since any scheme of finite type over a finite field has only finitely many points, there exists a repetition $\left(E_{i}, \theta_{i}\right) \simeq\left(E_{j}, \theta_{j}\right)$ with $j>i$. This gives periodicity of $\left(E_{i}, \theta_{i}\right)$.

In general, a slope semistable Higgs bundle with vanishing Chern classes do not need to be preperiodic. For example any non-torsion line bundle $L \in \operatorname{Pic}^{0}(X)$ with trivial Higgs field is not preperiodic. The second part of the above corollary is analogous to the fact that over $\overline{\mathbb{F}}_{p}$ strongly semistable vector bundles with vanishing Chern classes become étale trivializable after taking some Frobenius pull-back (see [4, Proposition 2.5]). This last fact can be also interpreted as saying that for $X / \overline{\mathbb{F}}_{p}$ Nori's fundamental group scheme is equal to the S-fundamental group scheme.

Let $M_{\text {Hodge }}^{0}(X, r)$ be the set of isomorphism classes of slope semistable systems of Hodge bundles of rank $r \leq p$ and with vanishing Chern classes. This set does not depend on the choice of ample polarization and it forms a bounded family. We can define the function $\Phi: M_{\text {Hodge }}^{0}(X, r) \rightarrow M_{\text {Hodge }}^{0}(X, r)$ which maps $(E, \theta)$ to the associated graded of Simpson's filtration of $C^{-1}(E, \theta)$. This defines a discrete dynamical system. Note that $(E, \theta)$ is periodic (preperiodic) if and only if the point of $M_{\text {Hodge }}^{0}(X, r)$ corresponding to $(E, \theta)$ is periodic (respectively, preperiodic) for the above defined dynamical system.

In the characteristic zero case one can also construct an analogous discrete dynamical system by taking the associated graded of Simpson's filtration of the flat bundle corresponding to a system of Hodge bundles via the KobayashiHitchin correspondence. However, in the characteristic zero case this system is less interesting as it carries much less information. 


\section{The Bogomolov-Miyaoka-Yau inequality in positive characteristic}

Let $X$ be a smooth projective surface over an algebraically closed field $k$ of characteristic $p$. Let us fix an ample divisor $H$ on $X$. Let $N^{1}(X)=\mathrm{NS}(X) \otimes \mathbb{R}$, where $\operatorname{NS}(X)$ is the Néron-Severi group of $X$. By the Hodge index theorem the intersection pairing on $N^{1}(X)$ has signature $(1, \rho-1)$, where $\rho=\operatorname{dim}_{\mathbb{R}} N^{1}(X)$. Therefore the open cone $D^{2}>0$ has two components separated by the hyperplane $D H=0$. Let $C^{+}$denote the positive component of this cone:

$$
C^{+}:=\left\{D \in N^{1}(X): D^{2}>0 \text { and } D H>0\right\} .
$$

The following proposition generalizes to arbitrary characteristic [34, Theorem 9], proven by Shepherd-Barron in characteristic 2:

Proposition 2 Let $X$ be a smooth projective surface that can be lifted to $W_{2}(k)$. Then $\Omega_{X}^{1}$ does not contain a line bundle $M$ with $M \in C^{+}$.

Proof Assume that $M \in C^{+}$and consider a system of Hodge bundles $(E:=$ $\left.E^{1} \oplus E^{0}, \theta\right)$ with $E^{1}=M, E^{0}=\mathscr{O}_{X}$ and $\theta: E^{1}=M \rightarrow E^{0} \otimes \Omega_{X}=\Omega_{X}$ given by the inclusion. Note that $(E, \theta)$ is slope $H$-stable since the only rank 1 Hodge subsystem of $(E, \theta)$ is of the form $\left(\mathscr{O}_{X}, 0\right)$. Therefore by Theorem 8 we have

$$
0=4 c_{2}(E) \geq c_{1}^{2}(E)=M^{2},
$$

a contradiction.

In [3, Theorem 4] Bogomolov proved that the cotangent bundle of a smooth complex projective surface does not contain big line bundles. Below we present an example showing that this is no longer true for smooth projective surfaces liftable to the Witt ring. So Proposition 2 is the best result one can expect in this situation.

Example 1 Let us recall the following example due to Ekedahl et al. (see [8, p. 23, Remark ii]). Let $X$ be a smooth projective Shimura surface, which is the moduli space of abelian 4-folds with multiplication by a totally indefinite quaternion algebra over a real quadratic field $F . X$ can be also obtained as a quotient of $\mathbb{H} \times \mathbb{H}$ by an irreducible arithmetic group (see [24, Example 4.4 and Example 5.6] for details). So $\Omega_{X}=L \oplus M$ where $L$ and $M$ are strictly nef line bundles with $L^{2}=M^{2}=0$ and $L M>0 . X$ is defined over $F$ and it has a smooth projective model $\mathscr{X} \rightarrow$ Spec $\mathscr{O}_{F, S}$, where $\mathscr{O}_{F, S}$ is a localization of the ring of integers of $F$ along a finite set of places $S$. For all the unramified prime ideals $\mathfrak{p} \subset \mathscr{O}_{F, S}$ we can base change $\mathscr{X}$ to the Witt ring $W(k)$ of the 
algebraic closure $k$ of $\mathscr{O}_{F, S} / \mathfrak{p}$. For all the rational primes $p$ that are inert in $F$ the reduction $L_{p} \subset \Omega_{X_{p}}$ of $L \subset \Omega_{X}$ is a big line bundle whereas $X_{p}$ lifts to the Witt ring of the algebraic closure of the residue field.

Corollary 7 Let $X$ be a smooth projective surface of non-negative Kodaira dimension. Assume that $X$ can be lifted to $W_{2}(k)$. Then $4 c_{2}(X) \geq c_{1}^{2}(X)$. Moreover, if $4 c_{2}(X)=c_{1}^{2}(X)$ then one of the following conditions is satisfied:

1. $K_{X}^{2}=0$,

2. $K_{X}^{2}>0$ and $p=2$. In this case $\Omega_{X}$ is slope stable with respect to every ample polarization.

Proof Let us note that if $X \rightarrow Y$ is a blow up at a smooth point and $X$ can be lifted to $W_{2}(k)$ then also $Y$ can be lifted to $W_{2}(k)$. Therefore, since blowing up a smooth surface increases $c_{2}$ and decreases $c_{1}^{2}$, we can assume that $X$ is minimal. In this case $K_{X}$ is nef and in particular $K_{X} \in \overline{C^{+}}$. If $\Omega_{X}$ is slope stable with respect to every ample polarization then the assertions follow from Corollary 2. So we can assume that $\Omega_{X}$ is not slope $H$-stable. Let $M$ be the maximal destabilizing subsheaf of $\Omega_{X}$. Since the quotient $\Omega_{X} / M$ is a rank 1 torsion free sheaf, we can write it as $I_{Z} L$, where $Z$ is a 0 -dimensional subscheme and $L$ is a line bundle such that $L+M=K_{X}$. By assumption we have $\left(2 M-K_{X}\right) H \geq 0$. Then

$$
c_{2}(X)=M L+\operatorname{deg} Z \geq M L=M\left(K_{X}-M\right) .
$$

If $\left(2 M-K_{X}\right)^{2}>0$ then $\left(2 M-K_{X}\right) \in C^{+}$and hence $2 M=\left(2 M-K_{X}\right)+$ $K_{X} \in C^{+}$, which contradicts Proposition 2. Therefore

$$
4 c_{2}(X)-c_{1}^{2}(X) \geq 4 M\left(K_{X}-M\right)-K_{X}^{2}=-\left(2 M-K_{X}\right)^{2} \geq 0,
$$

which proves the required inequality.

If $4 c_{2}(X)=c_{1}^{2}(X)>0$ then $K_{X} \in C^{+}$. So if $\Omega_{X}$ is not slope $H$-stable then $\left(2 M-K_{X}\right)^{2}=0$. This implies that $\left(2 M-K_{X}\right) \in \overline{C^{+}}$and hence $2 M=\left(2 M-K_{X}\right)+K_{X} \in C^{+}$, a contradiction. The fact that in this case $p=2$ follows from Theorem 13 .

Note that Shepherd-Barron proved the above inequality in case $p=2$ ([34, Theorem 9 and Corollary 11]) and he conjectured that it remains true in general (see [34, p. 244]). In fact, we show the following stronger version of this inequality:

Theorem 13 Let $X$ be a smooth projective surface of non-negative Kodaira dimension. Assume that $X$ can be lifted to $W_{2}(k)$. If $p \geq 3$ then $3 c_{2}(X) \geq$ $c_{1}^{2}(X)$. 
Proof As in the proof of Corollary 7 we can assume that $K_{X} \in \overline{C^{+}}$. There exists a canonical system of Hodge bundles $\left(E:=E^{1} \oplus E^{0}, \theta\right)$ with $E^{1}=\Omega_{X}$, $E^{0}=\mathscr{O}_{X}$ and $\theta:=\mathrm{id}_{\Omega_{X}}$. If $(E, \theta)$ is slope $H$-semistable then by Theorem 8

$$
3 c_{2}(E)=3 c_{2}(X) \geq c_{1}^{2}(E)=c_{1}^{2}(X) .
$$

So we can assume that $(E, \theta)$ is not slope $H$-semistable. Let $\left(E^{\prime}, \theta^{\prime}\right)$ be the maximal destabilizing Hodge subsystem. The only rank 1 Hodge subsystem is of the form $\left(\mathscr{O}_{X}, 0\right)$ and it does not destabilize $(E, \theta)$ so $E^{\prime}$ has rank 2 (and $\left(E^{\prime}, \theta^{\prime}\right)$ is slope $H$-stable). In this case $E^{\prime}=\mathscr{O}_{X} \oplus M$, where $M$ is a line bundle contained in $\Omega_{X}$. Since $\left(E^{\prime}, \theta^{\prime}\right)$ destabilizes $(E, \theta)$ we have

$$
\mu\left(E^{\prime}\right)=\frac{M H}{2}>\mu(E)=\frac{K_{X} H}{3} .
$$

Therefore $\left(3 M-2 K_{X}\right) H>0$. If $3 M-2 K_{X} \in C^{+}$then $3 M=\left(3 M-2 K_{X}\right)+$ $2 K_{X} \in C^{+}$, which contradicts Proposition 2 . This shows that $\left(3 M-2 K_{X}\right)^{2} \leq$ 0 .

As before we write $\Omega_{X} / M$ as $I_{Z} L$, where $Z$ is a 0 -dimensional subscheme and $L$ is a line bundle such that $L+M=K_{X}$. Then

$$
c_{2}(X)=M L+\operatorname{deg} Z \geq M L=M\left(K_{X}-M\right) .
$$

Therefore, since $M^{2} \leq 0$ by Proposition 2, we have

$3 c_{2}(X)-c_{1}^{2}(X) \geq 3 M\left(K_{X}-M\right)+\frac{3}{4} M^{2}-K_{X}^{2}=-\frac{1}{4}\left(3 M-2 K_{X}\right)^{2} \geq 0$.

The above proof of Theorem 13 follows quite closely proof of [35, Proposition 9.9] but in the dual setting.

Remark 3 Note that passing to Simpson's filtration can increase the level of nilpotence. This is the reason why proof of Theorem 13 does not work in characteristic 2 even though the level of nilpotence of the canonical system of Hodge bundles is only one.

Proposition 3 Under the assumptions of Theorem 13, the equality $3 c_{2}(X)=$ $c_{1}^{2}(X)$ holds if and only if $X$ is minimal and one of the following conditions is satisfied:

1. the canonical system of Hodge bundles $\left(E=\mathscr{O}_{X} \oplus \Omega_{X}, \theta\right)$ is slope $H$ stable and $\Delta(E)=0$,

2. $K_{X} \equiv 0$ and there exists a line subbundle $M \subset \Omega_{X}$ such that $M^{2}=0$ and $M H>0$ for an ample divisor $H$, 
3. $K_{X}^{2}=0, K_{X} \not \equiv 0$ and there exists a line subbundle $M \subset \Omega_{X}$ such that

$M \equiv a K_{X}$ for some positive rational number $a$,

4. $\Omega_{X}$ is numerically flat (in particular, $K_{X} \equiv 0$ ).

Proof Minimality of $X$ is clear. First consider the case in which $(E, \theta)$ is not slope $H$-semistable. Then the proof of Theorem 13 shows that $3 c_{2}(X)=c_{1}^{2}(X)$ implies that $M^{2}=\left(3 M-2 K_{X}\right)^{2}=0$ and $\operatorname{deg} Z=0$ (which implies that $M$ is a line subbundle of $\left.\Omega_{X}\right)$. Therefore $\left(3 M-2 K_{X}\right) \in \overline{C^{+}}$, which implies that $K_{X}^{2}=0$ (otherwise $K_{X} \in C^{+}$and $3 M=\left(3 M-2 K_{X}\right)+2 K_{X} \in C^{+}$, a contradiction). Hence $M K_{X}=0$ and by the Hodge index theorem either $K_{X} \equiv 0$ or $K_{X} \not \equiv 0$ and there exists some positive rational number $a$ such that $M \equiv a K_{X}$.

Now consider the case when $(E, \theta)$ is slope $H$-semistable but it is not slope $H$-stable. Then there exists a saturated slope $H$-stable subsystem of Hodge bundles $\left(E^{\prime}, \theta^{\prime}\right)$ such that $\mu\left(E^{\prime}\right)=\mu(E)$.

If $\left(E^{\prime}, \theta^{\prime}\right)=\left(\mathscr{O}_{X}, 0\right)$ then $K_{X} H=0$. So $K_{X}$ is numerically trivial by the Hodge index theorem. In this case the quotient $(E, \theta) /\left(E^{\prime}, \theta^{\prime}\right)=\left(\Omega_{X}, 0\right)$ is slope $H$-semistable. But then $\Omega_{X}$ is strongly slope $H$-semistable (see [26, Theorem 2.1]) and hence it is numerically flat (see [23, Proposition 5.1]).

If $E^{\prime}$ has rank 2 then $E^{\prime}=\mathscr{O}_{X} \oplus M$, where $M$ is a line bundle contained in $\Omega_{X}$. By assumption $\left(3 M-2 K_{X}\right) H=0$. The same arguments as before show that $M$ is a line subbundle of $\Omega_{X}$ and $M^{2}=\left(3 M-2 K_{X}\right)^{2}=0$. But then $3 M \equiv 2 K_{X}$, which also implies that $K_{X}^{2}=0$.

Remark 4 It is easy to see that cases 1,3 and 4 of the above proposition can occur in every characteristic (including characteristic zero). Assume that we are in the second case. Looking at the classification of surfaces one can easily see that $K_{X} \equiv 0$ and $c_{2}=0$ can occur only if $X$ is abelian or hyperelliptic or quasi-hyperelliptic. In the first two cases we have a short exact sequence

$$
0 \rightarrow \mathscr{O}_{X} \rightarrow \Omega_{X} \rightarrow \omega_{X} \rightarrow 0
$$

which easily gives a contradiction. This shows that $X$ is quasi-hyperelliptic. This can only occur in characteristics 2 and 3. Then we have a short exact sequence

$$
0 \rightarrow \mathscr{O}_{X}(m \Sigma) \rightarrow \Omega_{X} \rightarrow \omega_{X}(-m \Sigma) \rightarrow 0,
$$

where $\Sigma$ is the curve of cusps with $\Sigma^{2}=0$ and $m=2$ in characteristic 2 and $m=1$ in characteristic 3 . The above sequence implies that $M=\mathscr{O}_{X}(m \Sigma)$. There are examples of quasi-hyperelliptic surfaces with vanishing $H^{2}\left(T_{X}\right)$ (see [19, Theorem 4.2]). Since $H^{2}\left(T_{X}\right)$ is the obstruction space to lifting, such surfaces lift to $W_{2}(k)$. On the other hand, not all quasi-hyperelliptic surfaces 
lift to $W_{2}(k)$ as in some cases the Hodge to de Rham spectral sequence does not degenerate at $E_{1}$ (see [19, Theorem 4.4]) and we would get a contradiction with [6, Corollaire 2.4].

\section{Adjoint linear systems on surfaces}

Existence of Bogomolov's inequality for semistable sheaves on varieties liftable to $W_{2}$ has many interesting applications. In this subsection we state just two results on adjoint linear systems on surfaces. The first one generalizes Mumford's vanishing theorem (see [2, IV, Theorem 12.1]) and it was already known with a different proof (see [6, Corollaire 2.8]):

Theorem 14 Let $X$ be a smooth projective surface liftable to $W_{2}(k)$. Let $D$ be a nef and big line bundle on $X$. Then $H^{1}\left(X, K_{X}+D\right)=0$.

The vanishing follows immediately by replacing in proof of $[2$, IV, Theorem 12.1] usual Bogomolov's inequality with Corollary 2.

Note that the same arguments show a counterexample to Bogomolov's inequality for semistable sheaves in positive characteristic. More precisely, let $X$ be a smooth projective surface in positive characteristic for which Kodaira's vanishing fails (see [33] or [30]). Then there exists an ample divisor $H$ such that $H^{1}\left(X, K_{X}+H\right) \neq 0$. By Serre's duality $\operatorname{Ext} t^{1}\left(\mathscr{O}_{X}(H), \mathscr{O}_{X}\right) \neq 0$, so there exists a non-trivial extension

$$
0 \rightarrow \mathscr{O}_{X} \rightarrow E \rightarrow \mathscr{O}_{X}(H) \rightarrow 0 .
$$

Then the sheaf $E$ is slope $H$-semistable, but $\Delta(E)<0$.

Similarly to the above, the usual characteristic zero proof can be used to show the following Reider's type theorem (cf. [2, IV, Section 11]). Details are left to the reader.

Theorem 15 Let $X$ be a smooth projective surface liftable to $W_{2}(k)$. Let $D$ be a nef line bundle on $X$ and let $Z$ be a zero-dimensional locally complete intersection subscheme of $X$. Assume that $D^{2}>4 \operatorname{deg} Z$. Then $Z$ is in special position with respect to $K_{X}+D$ if and only if there there exists an effective curve $C$ containing $Z$ such that $Z$ is in (very) special position with respect to $\mathscr{O}_{C}\left(K_{X}+D\right)$. Moreover, we can choose $C$ so that $D-2 C$ is big, $D C-\operatorname{deg} Z \leq$ $C^{2}$ and $0 \leq D C<2 \operatorname{deg} Z$.

The above theorem has many applications to studying $n$-very ampleness and $n$-jet ampleness of adjoint linear systems, to pluricanonical maps on surfaces of general type, linear systems on surfaces of Kodaira dimension 0, etc. 


\section{Lifting of Frobenius split varieties}

Let $f: X \rightarrow S$ be a flat morphism of schemes in characteristic $p$ and let $F_{X / S}: X \rightarrow X^{\prime}$ be the relative Frobenius morphism. Let us fix a flat $\mathbb{Z} / p^{2} \mathbb{Z}$ scheme $\tilde{S}$ such that $S \rightarrow \tilde{S}$ is the closed subscheme defined by $p$. Then $p \mathscr{O}_{\tilde{S}} \simeq \mathscr{O}_{S}$.

We say that an $S$-scheme $X / S$ is $F$-split is the canonical map $\mathscr{O}_{X^{\prime}} \rightarrow$ $F_{X / S, *} \mathscr{O}_{X}$ can be split by some $\mathscr{O}_{X^{\prime}}$-linear map $F_{X / S, *} \mathscr{O}_{X} \rightarrow \mathscr{O}_{X^{\prime}}$.

The following proposition was pointed out to the author by Bhargav Bhatt:

Proposition 4 If $X / S$ is F-split then $X^{\prime}$ can be lifted to $\tilde{S}$.

Proof Let $L_{X / S}$ be the cotangent complex of $X / S$. By definition of the cotangent complex it is easy to see that the map $F_{X / S}^{*} L_{X^{\prime} / S} \rightarrow L_{X / S}$ induced by the relative Frobenius morphism is the zero map.

There exists a canonical obstruction $o b_{X / S}: L_{X / S} \rightarrow \mathscr{O}_{X}[2]$ to lifting $X / S$ to $\tilde{S}$ (see [13, Chapitre III, Théorème 2.1.7]). By functoriality of obstructions we have the following commutative diagram

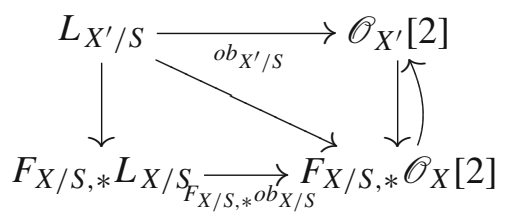

But the vertical map $L_{X^{\prime} / S} \rightarrow F_{X / S, *} L_{X / S}$ is adjoint to $F_{X / S}^{*} L_{X^{\prime} / S} \rightarrow L_{X / S}$, so it is zero. This implies that $o b_{X^{\prime} / S}=0$ and $X^{\prime}$ can be lifted to $\tilde{S}$.

In the smooth case this proposition was proven in [14, p. 164] (see also [16, Corollary 9.2]).

Let us recall that $X / S$ is $F$-pure if the Frobenius map $\mathscr{O}_{X^{\prime}} \rightarrow F_{X / S, *} \mathscr{O}_{X}$ is locally split as a map of $\mathscr{O}_{X^{\prime}}$-modules. Proposition 4 immediately implies the following corollary:

Corollary 8 If $X / S$ is F-pure then locally in the Zariski topology X can be lifted modulo $p^{2}$.

Together with Theorem 8 , Proposition 4 implies also the following corollary:

Corollary 9 Let $X$ be a smooth projective variety defined over an algebraically closed field of positive characteristic $p$. Let $H$ be an ample divisor on $X$. Assume that $X$ is $F$-split. Then for any slope $H$-semistable Higgs sheaf $(E, \theta)$ on $X$ of rank $r \leq p$ we have $\Delta(E) H^{n-2} \geq 0$. 
Acknowledgments The author would like to thank Bhargav Bhatt for allowing him to include Proposition 4 and Yanhong Yang for pointing out a problem with the first proof of Lemma 5. Author's work was partially supported by Polish National Science Centre (NCN) contract number 2012/07/B/ST1/03343.

Open Access This article is distributed under the terms of the Creative Commons Attribution License which permits any use, distribution, and reproduction in any medium, provided the original author(s) and the source are credited.

\section{References}

1. Anchouche, B.: Bogomolov inequality for Higgs parabolic bundles. Manuscripta Math. 100, 423-436 (1999)

2. Barth, W., Hulek, K., Peters, C., Van de Ven, A.: Compact Complex Surfaces, 2nd edn. Ergebnisse der Mathematik und ihrer Grenzgebiete, vol. 4, 3 Folge. Springer, Berlin (2004)

3. Bogomolov, F.A.: Holomorphic tensors and vector bundles on projective varieties. Izv. Akad. Nauk SSSR 42, 1227-1287 (1978)

4. Brenner, H., Kaid, A.: On deep Frobenius descent and flat bundles. Math. Res. Lett. 15, 1101-1115 (2008)

5. Bruzzo, U., Hernández Ruipérez, D.: Semistability vs. nefness for (Higgs) vector bundles. Differ. Geom. Appl. 24, 403-416 (2006)

6. Deligne, P., Illusie, L.: Relévements modulo $p^{2}$ et décomposition du complexe de de Rham. Invent. Math. 89, 247-270 (1987)

7. Easton, R.W.: Surfaces violating Bogomolov-Miyaoka-Yau in positive characteristic. Proc. Am. Math. Soc. 136, 2271-2278 (2008)

8. Ekedahl, T., Shepherd-Barron, N., Taylor, R.: A conjecture on the existence of compact leaves of algebraic foliations (1999). https://www.dpmms.cam.ac.uk/ nisb/

9. Faltings, G.: Crystalline cohomology and $p$-adic Galois-representations. Algebraic Analysis, Geometry, and Number Theory (Baltimore, MD, 1988), pp. 25-80. Johns Hopkins Univ. Press, Baltimore (1989)

10. Ferrand, D.: Descente de la platitude par un homomorphisme fini. C. R. Acad. Sci. Paris Sér. A-B 269, A946-A949 (1969)

11. Gieseker, D.: On a theorem of Bogomolov on Chern classes of stable bundles. Am. J. Math. 101, 77-85 (1979)

12. Huybrechts, D., Lehn, M.: The Geometry of Moduli Spaces of Sheaves. Aspects of Mathematics, vol. 31, xiv+269 pp. Friedrich Vieweg \& Sohn, Braunschweig (1997)

13. Illusie, L.: Complexe Cotangent et Déformations. I. Lecture Notes in Mathematics, vol. 239. Springer, Berlin (1971)

14. Illusie, L.: Frobenius et dégénérescence de Hodge. In: Introduction la théorie de Hodge, pp. 113-168. Panoramas et Synthèses, vol. 3. Soc. Math. France, Paris (1996)

15. Jang, J.: Generically ordinary fibrations and a counterexample to Parshin's conjecture. Mich. Math. J. 59, 169-178 (2010)

16. Joshi, K.: Exotic torsion, Frobenius splitting and the slope spectral sequence. Can. Math. Bull. 50, 567-578 (2007)

17. Lan, G., Sheng, M., Yang, Y., Zuo, K.: Semistable Higgs bundles of small ranks are strongly Higgs semistable (2013). arXiv:1311.2405v1

18. Lan, G., Sheng, M., Zuo, K.: Semistable Higgs bundles and representations of algebraic fundamental groups: positive characteristic case (2012). arXiv:1210.8280v1

19. Lang, W.: Quasi-elliptic surfaces in characteristic three. Ann. Sci. École Norm. Sup. 12, 473-500 (1979) 
20. Langer, A.: A Note on Bogomolov's Instability and Higgs Sheaves. Algebraic Geometry, pp. 237-256. de Gruyter, Berlin (2002)

21. Langer, A.: Semistable sheaves in positive characteristic. Ann. Math. 159, 241-276 (2004)

22. Langer, A.: Moduli spaces of sheaves in mixed characteristic. Duke Math. J. 124, 571-586 (2004)

23. Langer, A.: On the $S$-fundamental group scheme. Ann. Inst. Fourier 61, 2077-2119 (2011)

24. Langer, A. : On positivity and semistability of vector bundles in finite and mixed characteristics. J. Ramanujan Math. Soc. 28A(Special Issue), 287-309 (2013)

25. Langer, A.: Semistable modules over Lie algebroids in positive characteristic. Doc. Math. 19, 509-540 (2014)

26. Mehta, V.B., Ramanathan, A.: Homogeneous Bundles in Characteristic p. Algebraic Geometry-Open Problems (Ravello, 1982). Lecture Notes in Mathematics, vol. 997, pp. 315-320 (1983)

27. Miyaoka, Y.: On the Chern numbers of surfaces of general type. Invent. Math. 42, 225-237 (1977)

28. Mochizuki, T.: Kobayashi-Hitchin correspondence for tame harmonic bundles and an application. Astérisque 309 viii+117 pp. (2006)

29. Mochizuki, T.: Kobayashi-Hitchin correspondence for tame harmonic bundles. II. Geom. Topol. 13, 359-455 (2009)

30. Mukai, S.: Counterexamples to Kodaira's vanishing and Yau's inequality in positive characteristics. Kyoto J. Math. 53, 515-532 (2013)

31. Ogus, A., Vologodsky, V.: Nonabelian Hodge theory in characteristic p. Publ. Math. Inst. Hautes Études Sci. 106, 1-138 (2007)

32. Ramanan, S., Ramanathan, A.: Some remarks on the instability flag. Tohoku Math. J. 36, 269-291 (1984)

33. Raynaud, M.: Contre-exemple au "vanishing theorem" en caractéristique $p>0$, C. P. Ramanujam-a tribute, pp. 273-278. Tata Institute of Fundamental Research Studies in Mathematics, vol. 8. Springer, Berlin (1978)

34. Shepherd-Barron, N.: Unstable vector bundles and linear systems on surfaces in characteristic $p$. Invent. Math. 106, 243-262 (1991)

35. Simpson, C.: Constructing variations of Hodge structure using Yang-Mills theory and applications to uniformization. J. Am. Math. Soc. 1, 867-918 (1988)

36. Simpson, C.: Higgs bundles and local systems. Inst. Hautes Études Sci. Publ. Math. 75, 5-95 (1992)

37. Szpiro, L.: Sur le théorème de rigidité de Parsin et Arakelov. Journées de Géométrie Algébrique de Rennes Astérisque 64, 169-202

38. Yau, S.T.: Calabi's conjecture and some new results in algebraic geometry. Proc. Nat. Acad. Sci. USA 74, 1798-1799 (1977) 